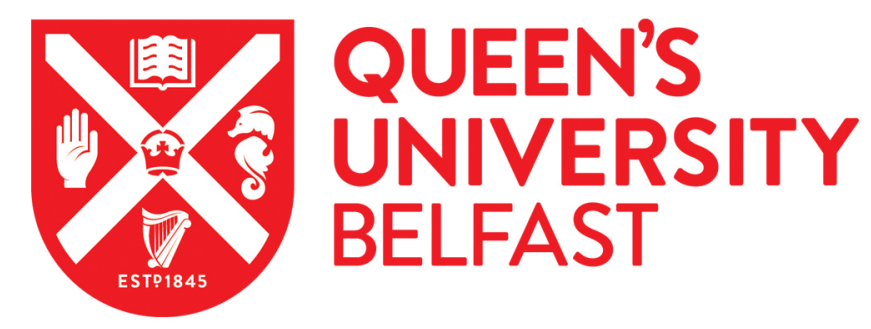

\title{
GIANT LOBES OF CENTAURUS A RADIO GALAXY OBSERVED WITH THE SUZAKU X-RAY SATELLITE
}

Stawarz, L., Tanaka, Y. T., Madejski, G., O'Sullivan, S. P., Cheung, C. C., Feain, I. J., Fukazawa, Y., Gandhi, P., Hardcastle, M. J., Kataoka, J., Ostrowski, M., Reville, B., Siemiginowska, A., Simionescu, A., Takahashi, T., Takei, Y., Takeuchi, Y., \& Werner, N. (2013). GIANT LOBES OF CENTAURUS A RADIO GALAXY OBSERVED WITH THE SUZAKU X-RAY SATELLITE. Astrophysical Journal, 766(1), [ARTN 48].

https://doi.org/10.1088/0004-637X/766/1/48

Published in:

Astrophysical Journal

Document Version:

Publisher's PDF, also known as Version of record

Queen's University Belfast - Research Portal:

Link to publication record in Queen's University Belfast Research Portal

Publisher rights

Copyright 2013. The American Astronomical Society. All rights reserved. This work is made available online in accordance with the publisher's policies. Please refer to any applicable terms of use of the publisher.

\section{General rights}

Copyright for the publications made accessible via the Queen's University Belfast Research Portal is retained by the author(s) and / or other copyright owners and it is a condition of accessing these publications that users recognise and abide by the legal requirements associated with these rights.

Take down policy

The Research Portal is Queen's institutional repository that provides access to Queen's research output. Every effort has been made to ensure that content in the Research Portal does not infringe any person's rights, or applicable UK laws. If you discover content in the Research Portal that you believe breaches copyright or violates any law, please contact openaccess@qub.ac.uk. 


\title{
GIANT LOBES OF CENTAURUS A RADIO GALAXY OBSERVED WITH THE SUZAKU X-RAY SATELLITE
}

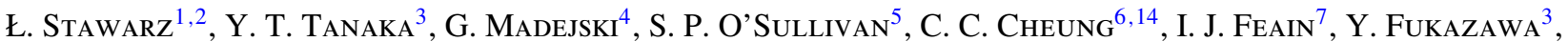

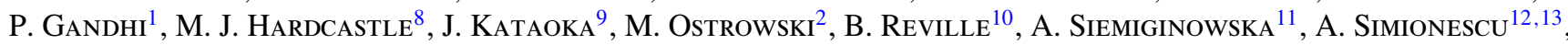 \\ T. TAKAHASHI ${ }^{1}$, Y. TAKEI $^{1}$, Y. TAKEUCHI $^{9}$, AND N. WERNER $^{12,13}$ \\ ${ }^{1}$ Institute of Space and Astronautical Science JAXA, 3-1-1 Yoshinodai, Chuo-ku, Sagamihara, Kanagawa 252-5210, Japan; stawarz@ astro.isas.jaxa.jp \\ ${ }^{2}$ Astronomical Observatory, Jagiellonian University, ul. Orla 171, 30-244 Kraków, Poland \\ ${ }^{3}$ Department of Physical Sciences, Hiroshima University, Higashi-Hiroshima, Hiroshima 739-8526, Japan \\ ${ }^{4}$ W. W. Hansen Experimental Physics Laboratory, Kavli Institute for Particle Astrophysics and Cosmology, Department of Physics \\ and SLAC National Accelerator Laboratory, Stanford University, Stanford, CA 94305, USA \\ ${ }^{5}$ Sydney Institute for Astronomy, School of Physics A28, University of Sydney, Sydney, NSW 2006, Australia \\ ${ }^{6}$ National Academy of Sciences, Washington, DC 20001, USA \\ ${ }^{7}$ CSIRO Astronomy and Space Science, ATNF, P.O. Box 76, Epping, NSW 1710, Australia \\ ${ }^{8}$ School of Physics, Astronomy and Mathematics, University of Hertfordshire, College Lane, Hatfield AL10 9AB, UK \\ ${ }^{9}$ Research Institute for Science and Engineering, Waseda University, 3-4-1, Okubo, Shinjuku, Tokyo 169-8555, Japan \\ ${ }^{10}$ Clarendon Laboratory, University of Oxford, Parks Road, Oxford OX1 3PU, UK \\ ${ }^{11}$ Harvard Smithsonian Center for Astrophysics, 60 Garden Street, Cambridge, MA 02138, USA \\ ${ }^{12}$ KIPAC, Stanford University, 452 Lomita Mall, Stanford, CA 94305, USA \\ ${ }^{13}$ Department of Physics, Stanford University, 382 Via Pueblo Mall, Stanford, CA 94305-4060, USA \\ Received 2012 October 15; accepted 2013 January 29; published 2013 March 6
}

\begin{abstract}
We report on Suzaku observations of selected regions within the southern giant lobe of the radio galaxy Centaurus A. In our analysis we focus on distinct X-ray features detected with the X-ray Imaging Spectrometer within the range $0.5-10 \mathrm{keV}$, some of which are likely associated with fine structure of the lobe revealed by recent high-quality radio intensity and polarization maps. With the available photon statistics, we find that the spectral properties of the detected X-ray features are equally consistent with thermal emission from hot gas with temperatures $k T>1 \mathrm{keV}$, or with a power-law radiation continuum characterized by photon indices $\Gamma \sim 2.0 \pm 0.5$. However, the plasma parameters implied by these different models favor a synchrotron origin for the analyzed X-ray spots, indicating that a very efficient acceleration of electrons up to $\gtrsim 10 \mathrm{TeV}$ energies is taking place within the giant structure of Centaurus A, albeit only in isolated and compact regions associated with extended and highly polarized radio filaments. We also present a detailed analysis of the diffuse X-ray emission filling the whole field of view of the instrument, resulting in a tentative detection of a soft excess component best fitted by a thermal model with a temperature of $k T \sim 0.5 \mathrm{keV}$. The exact origin of the observed excess remains uncertain, although energetic considerations point to thermal gas filling the bulk of the volume of the lobe and mixed with the non-thermal plasma, rather than to the alternative scenario involving a condensation of the hot intergalactic medium around the edges of the expanding radio structure. If correct, this would be the first detection of the thermal content of the extended lobes of a radio galaxy in X-rays. The corresponding number density of the thermal gas in such a case is $n_{g} \sim 10^{-4} \mathrm{~cm}^{-3}$, while its pressure appears to be in almost exact equipartition with the volume-averaged nonthermal pressure provided by the radio-emitting electrons and the lobes' magnetic field. A prominent large-scale fluctuation of the Galactic foreground emission, resulting in excess foreground X-ray emission aligned with the lobe, cannot be ruled out. Although tentative, our findings potentially imply that the structure of the extended lobes in active galaxies is likely to be highly inhomogeneous and non-uniform, with magnetic reconnection and turbulent acceleration processes continuously converting magnetic energy to internal energy of the plasma particles, leading to possibly significant spatial and temporal variations in the plasma $\beta$ parameter around the volume-averaged equilibrium condition $\beta \sim 1$.
\end{abstract}

Key words: galaxies: active - galaxies: individual (Centaurus A) - galaxies: jets - intergalactic medium - magnetic fields - X-rays: galaxies

Online-only material: color figures

\section{INTRODUCTION}

The radio source Centaurus A is hosted by a massive elliptical galaxy NGC 5128, located at a distance of $D=3.7 \mathrm{Mpc}$ (for a review see Israel 1998). As such, it is the closest active galaxy, and its innermost structure, down to hundreds of Schwarzschild radii of the central black hole (mass $M_{\mathrm{BH}} \simeq 10^{8} M_{\odot}$; Neumayer 2010), can be studied in great detail with modern high-resolution

\footnotetext{
${ }^{14}$ National Research Council Research Associate; resident at Naval Research
} Laboratory, Washington, DC 20375, USA. and high-sensitivity instruments. Yet Centaurus A is at the same time surrounded by a giant radio halo, or rather giant lobes, extending for $\sim 600 \mathrm{kpc}$ in the north-south direction and $\sim 200 \mathrm{kpc}$ wide. This fossil structure, although it has been known for a while (Cooper et al. 1965), is still relatively poorly studied, because of its huge angular size $(\sim 5 \mathrm{deg} \times 9 \mathrm{deg})$, which in the past has precluded detailed observational studies. This situation has changed only very recently, and the giant lobes of the Centaurus A system can now be investigated with the improved angular resolution available at radio and $\gamma$-ray frequencies. 
Since its discovery, the giant halo of Centaurus A (total radio luminosity $L_{\mathrm{R}} \simeq 10^{41} \mathrm{erg} \mathrm{s}^{-1}$ ) has been observed multiple times with low-resolution single-dish and space-borne radio telescopes. Detailed analysis indicated that the radio spectrum of the southern lobe steepens systematically both with frequency and with distance from the core, in agreement with the expectation for an aging relic ( $\sim 30$ Myr old) system with the volume-averaged equipartition magnetic field $B_{\mathrm{eq}} \simeq 1.3 \mu \mathrm{G}$. Meanwhile, the radio spectrum of the northern lobe was found to be roughly position-independent, inconsistent with the scenario of passive aging, but suggesting instead an ongoing or at least recent injection of radiating electrons throughout the entire volume of the lobe (Hardcastle et al. 2009). The most recent high-resolution radio observations of the giant structure at $1.4 \mathrm{GHz}$ with ATCA and Parkes $64 \mathrm{~m}$ telescopes revealed a very inhomogeneous and filamentary structure of the lobes (Feain et al. 2011). The 50" resolution map derived from this study - "the most detailed radio continuum image of any radio galaxy to date"-showed that the northern lobe constitutes an extension of the inner structure (down to the radio core), for which the outer part could be resolved into a series of semiregularly spaced $(\simeq 25 \mathrm{kpc})$ and concentric shells or filaments with a projected thickness of 3-6 kpc, particularly prominent in the high-resolution radio polarized intensity maps. By contrast, the southern lobe, which appears to be physically unconnected to the core, displayed a largely chaotic and mottled morphology.

The magnetic field structure and thermal matter content of the giant lobes in Centaurus A were also probed recently at radio frequencies by an analysis of the Faraday rotation measure (RM) using the ensemble of polarized background sources (Feain et al. 2009). The RM analysis showed that the polarized emission in the northern lobe follows closely the continuum emission down to the sensitivity limits of the survey, with little evidence for depolarization, whereas the emission in the southern lobe is depolarized and chaotic with large jumps in the polarization angle. The apparent lack of internal depolarization effects for most of the giant structure enabled a meaningful limit to be placed on the volume-averaged density of the thermal plasma within the lobes $\bar{n}_{g}<0.5 \times 10^{-4}\left(B / B_{\text {eq }}\right)^{-1} \mathrm{~cm}^{-3}$. This limit is not inconsistent with the analysis of the improved RM data presented in O'Sullivan et al. (2013), resulting in a positive detection of the internal depolarization signal, with the corresponding gas density being $\bar{n}_{g} \sim 10^{-4} \mathrm{~cm}^{-3}$. The revealed non-uniform distribution of the RM excess throughout the entire volume of the source should, however, be kept in mind, as it reflects a highly inhomogeneous distribution of the thermal gas mixed with the lobes, and/or a complex topology of the lobes' magnetic field.

Centaurus $\mathrm{A}$ is a source of $\gamma$-ray emission within the broad energy range from $\gtrsim 50 \mathrm{keV}$ up to $\lesssim 10 \mathrm{TeV}$, as established by the pioneering observations with Compton Gamma-Ray Observatory (Steinle et al. 1998) and H.E.S.S. Cerenkov Telescope (Aharonian et al. 2009). It was widely believed, however, that it is the active core (nucleus and nuclear jet) that dominates the production of $\gamma$-rays in the system, especially because this core appears as a very prominent X-ray emitter with a complex, multi-component spectrum (see, e.g., Fukazawa et al. 2011, and references therein). And, indeed, even the most recent analysis of the INTEGRAL/SPI data resulted in only upper limits for the soft $\gamma$-ray emission of the giant lobes $(0.04-1 \mathrm{MeV}$ photon flux $<1.1 \times 10^{-3} \mathrm{ph} \mathrm{cm}^{-2} \mathrm{~s}^{-1}$; Beckmann et al. 2011). On the other hand, during the first 10 months of its operation, the new-generation $\gamma$-ray satellite Fermi-LAT resolved the giant lobes of Centaurus A (Abdo et al. 2010), revealing that they contribute about half of the total flux of the whole system at $\mathrm{MeV} / \mathrm{GeV}$ photon energies (the $0.1-100 \mathrm{GeV}$ lobes' luminosity is $L_{\gamma} \simeq 10^{41} \mathrm{erg} \mathrm{s}^{-1}$ ). This important result proved that $\gamma$-rays are being efficiently generated within the extended halo, despite the advanced age and relaxed nature of the structure. ${ }^{15}$ The detected emission, well modeled as inverse-Compton (IC) upscattering of cosmic background photons by ultrarelativistic electrons, moreover implies that the giant lobes in Centaurus A are close to the condition of energy/pressure equipartition between the radiating particles and the magnetic field (volumeaveraged field strength $\bar{B} \simeq 0.9 \mu \mathrm{G} \lesssim B_{\text {eq }}$ ).

Despite these new data, our understanding of the giant lobes in Centaurus A is still incomplete. Particularly missing in this context is X-ray information, even though the largescale structure of Centaurus A has been the subject of lowresolution, low-sensitivity observations with several previous $\mathrm{X}$-ray satellites. The first meaningful upper limits for the $\mathrm{X}$-ray emission of the giant lobes in the system were provided by the SAS 3 instrument (Marshall \& Clark 1981), which probed the $2-10 \mathrm{keV}$ continuum of the target down to a flux level of $3 \times 10^{-11} \mathrm{erg} \mathrm{cm}^{-2} \mathrm{~s}^{-1}$ (90\% limit). Subsequent observations with ROSAT suggested the presence of a giant X-ray structure around the radio halo (Arp 1994), which was, however, not confirmed by later analysis. Finally, ASCA observations of the outer part of the northern lobe revealed the presence of a compact X-ray feature with the 2-10 keV flux $\sim 10^{-12} \mathrm{erg} \mathrm{cm}^{-2} \mathrm{~s}^{-1}$, which was modeled as a power-law continuum or equivalently by thermal emission from a very hot gas $(k T \sim 10 \mathrm{keV}$; Isobe et al. 2001). In addition, a soft diffuse emission component with a total $0.5-2 \mathrm{keV}$ flux $\sim 8.5 \times 10^{-14} \mathrm{erg} \mathrm{cm}^{-2} \mathrm{~s}^{-1} / 0.55 \mathrm{deg}^{2}$, distributed within the whole field of view (FOV) of the GIS detector, best fitted by a thermal model with $k T \sim 0.6 \mathrm{keV}$, was tentatively found. Our preliminary analysis of the archival $A S C A$ data confirmed the presence of the hard X-ray spot, consisting of several intensity peaks aligned roughly with a highly polarized radio filament. However, some of the X-ray emission peaks coincide with the positions of optical background radio sources and as such may be unrelated to the giant lobes. A more detailed discussion on the archival $A S C A$ data, together with the analysis of the forthcoming follow-up Suzaku and Chandra observations, will be published in a later paper.

To close the gap between the X-ray studies of the giant halo in Centaurus $\mathrm{A}$ and the most recent observations at other wavelengths, in previous years we have requested the very first Suzaku observations of the southern lobe (AO-5, 51401). The low and relatively constant instrumental background of Suzaku is ideally suited for observations of low surface brightness diffuse sources such as giant lobes of Centaurus A. In addition, the spatial resolution of Suzaku's X-ray Imaging Spectrometer (XIS, $\simeq 2^{\prime}$ ) is well matched to the recently obtained highresolution $\left(50^{\prime \prime}\right)$ radio maps of the giant lobes, while the FOV of the instrument $(\simeq 1 / 3 \mathrm{deg} \times 1 / 3 \mathrm{deg})$ is large enough to encapsulate within a single Suzaku pointing the large-scale structures (shells/filaments) revealed by the radio polarization maps; Suzaku is therefore an ideal instrument for this work. However, because of the large extension of the giant lobes on the sky, only a small part of their structure could be covered in the two $80 \mathrm{ks}$ exposures that were performed. In this paper we report on the analysis of these Suzaku data, focusing first on distinct

\footnotetext{
15 See also in this context Takeuchi et al. (2012) and Katsuta et al. (2013) for the cases of two other radio galaxies with the extended lobes possibly resolved or at least detected by Fermi-LAT.
} 


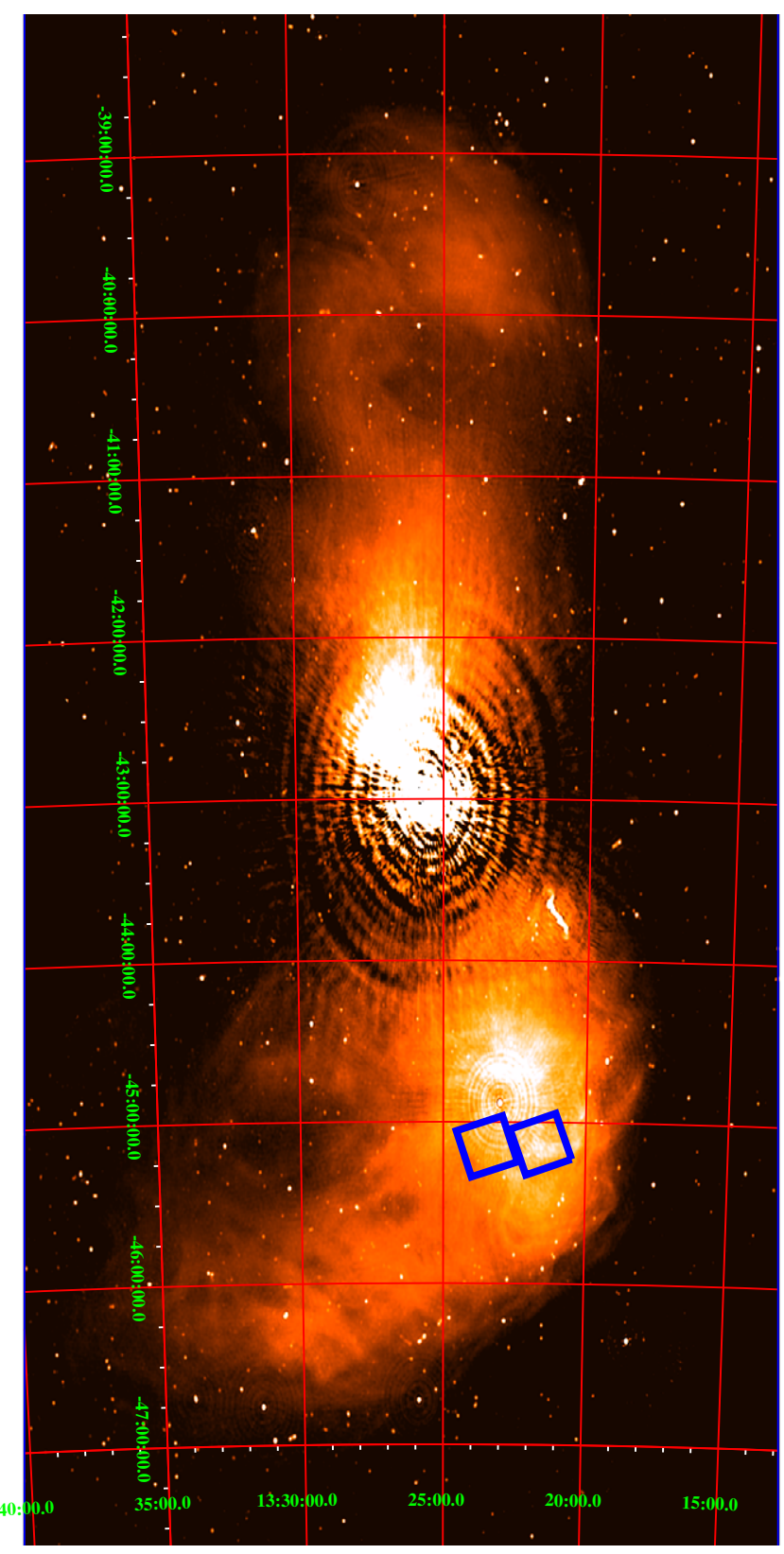

Figure 1. 1.4 GHz total intensity map of the giant Centaurus A structure (Feain et al. 2011) with the two Suzaku "on" pointings marked as blue squares (see Table 1).

(A color version of this figure is available in the online journal.)

X-ray features detected within the FOV of the XIS centered on the radio-bright part of the southern lobe, and second on the diffuse emission filling the whole FOV of the instrument and apparently related to the Centaurus A halo. Our results are presented in detail in Section 2 and discussed further in Section 3.

\section{SUZAKU DATA}

\subsection{Observations and Data Processing}

Two regions within the southern lobe of Centaurus A were observed with the Suzaku satellite (Mitsuda et al. 2007) from 2010 August 10 to 12, as indicated in Figure 1, showing the $1.4 \mathrm{GHz}$ total intensity map of the whole giant Centaurus A structure (Feain et al. 2011), with the two Suzaku pointings marked as blue squares. The observation log, with details such as the nominal pointing positions and observation times, is summarized in the first two rows of Table 1 ("Lobe 1" and "Lobe 2" exposures). In this paper, we restrict the analysis to the data taken with the XIS instrument (Koyama et al. 2007), which consists of four CCD sensors each placed on the focal plane of the X-Ray Telescope (Serlemitsos et al. 2007), and focus first (Sections 2.2 and 2.3) on faint X-ray features likely associated, as argued below, with fine structure of the lobe revealed by the most recent high-quality radio maps (Feain et al. 2011). Next (Section 2.4) we present the analysis of the diffuse emission component filling the whole FOV of the instrument; for this analysis we estimate the background from two shorter "off" Suzaku exposures, targeting the blank sky outside of (but close to) the Centaurus A southern lobe, as summarized in the last two rows of Table 1 ("Lobe 3" and "Lobe 4"; see also Figure 7 below). At the time of the performed observations the two frontilluminated CCDs (XIS0 and XIS3) and one back-illuminated CCD (XIS1) were working well, so the data discussed below for all the pointings were performed with all the XIS sensors set to full-window and normal clocking modes.

In the following we describe the screening criteria for XIS data. Events with GRADE 0,2, 3, 4, and 6 were utilized in the data reduction procedure, and flickering pixels were removed by using cleansis. We selected good-time intervals by the same criteria as described in the The Suzaku Data Reduction Guide ${ }^{16}$ SAA_HXD $==0$ \&\& T_SAA_HXD $>436 \& \& E L V>5$ $\& \& \quad$ DYE_ELV $>2 \quad \& \&$ ANG_DIST $<1.5 \quad \& \& \quad$ SO_DTRATE $<3$ $\& \& A O C U_{-H K}$ CNT3_NML_P $==1$. In the analysis we used the HEADAS software version 6.11 and calibration database (CALDB) released on 2012 February 11. The net exposures were $75.2 \mathrm{ks}$ and $81.4 \mathrm{ks}$ for the "on" pointings Lobe 1 and Lobe 2, respectively, and about $20 \mathrm{ks}$ for the "off" pointings Lobe 3 and Lobe 4.

\subsection{Compact $X$-Ray Features}

Figure 2 presents the Suzaku image of the targeted "on" regions within the Centaurus A southern lobe, corresponding to the photon energy range $0.5-10 \mathrm{keV}$, with cataloged background or foreground sources listed in the SIMBAD database marked by green crosses. The figure corresponds to the combined XISO and XIS3 images after the subtraction of the "non-X-ray background" (NXB) and the vignetting correction (based on a flat image produced using xissim; Ishisaki et al. 2007). The maps were binned by a factor of eight in each $(x, y)$ direction and smoothed with a Gaussian kernel of four pixels. The NXB images were obtained from the night Earth data using xisnxbgen and corrected for the exposure using xi sexpmapgen (Tawa et al. 2008).

As shown in the images, several bright X-ray spots are present within the FOV of the XIS instrument. The positions of three prominent spots closely match the positions of point-like optical objects identified as stars: HD 116386 at (R.A. = 201.015, decl. $=-45.2373)$, HD 116335 at $(200.9349,-45.1980)$, and HD 116099 at $(200.5186,-45.0564)$, together with nearby TYC 8248-981-1 at (200.5203, -45.0441); these three X-ray features are therefore considered here as most likely unrelated to the lobe. We note that X-ray counterparts for HD 116386 and HD 116099 are listed in the ROSAT All-Sky Survey catalog as 1RXS J132403.1-451358 and 1RXS J132204.7-450312, respectively; these, in fact, are the only cataloged X-ray sources

\footnotetext{
16 http://heasarc.nasa.gov/docs/suzaku/analysis/abc/
} 


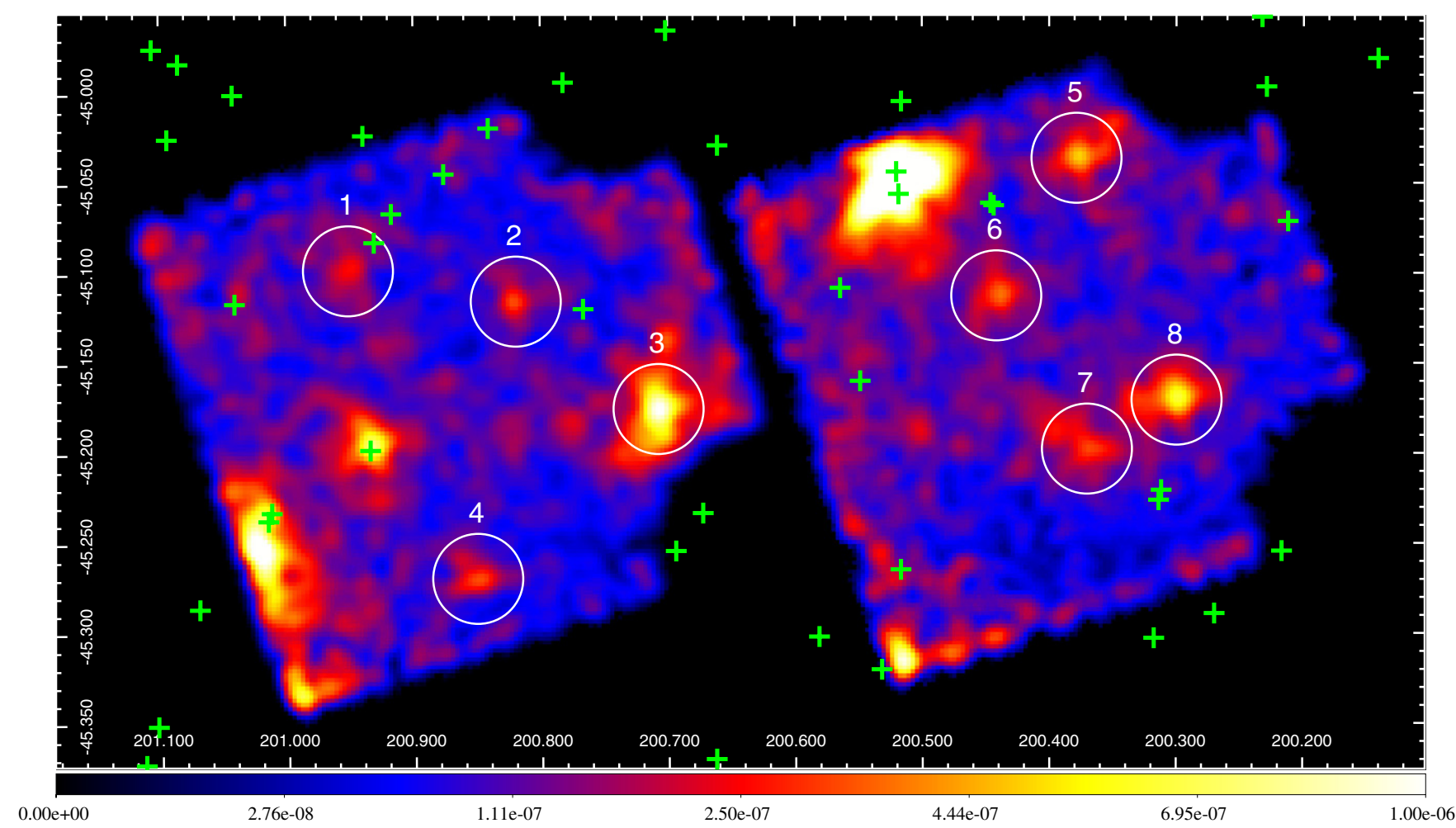

Figure 2. Suzaku XIS0+3 image (after vignetting correction and subtraction of the NXB) of the targeted regions within the Centaurus A southern lobe corresponding to the photon energy range $0.5-10 \mathrm{keV}$. Green crosses mark the positions of the foreground/background sources from the SIMBAD database. White circles mark the flux extraction regions for the X-ray features that we consider as "possibly related to the lobe." Spurious X-ray features are present in the bottom-left corners of the two FOVs, resulting from instrumental artifacts.

(A color version of this figure is available in the online journal.)

Table 1

Suzaku Observations of Centaurus A in 2010

\begin{tabular}{|c|c|c|c|c|c|}
\hline Target & Obs. ID & (R.A., Decl.) & $\begin{array}{l}\text { Start time } \\
\text { (UT) }\end{array}$ & $\begin{array}{l}\text { End time } \\
\text { (UT) }\end{array}$ & Net Exposure \\
\hline Lobe 1 (“on”) & 705032010 & $\left(200.867,-45^{\circ} .151\right)$ & Aug 10 15:52:16 & Aug 11 18:37:10 & 75.2 \\
\hline Lobe 2 (“on”) & 705033010 & $(200.394,-45.136)$ & Aug 11 18:38:19 & Aug 12 23:08:19 & 81.4 \\
\hline Lobe 3 (“off”) & 705034010 & $(205.750,-45.132)$ & Aug 12 23:12:20 & Aug 13 08:17:12 & 20.1 \\
\hline Lobe 4 (“off”) & 705035010 & $(196.750,-45.136)$ & Aug 13 08:21:01 & Aug 13 16:10:12 & 20.7 \\
\hline
\end{tabular}

Note. ${ }^{\mathrm{a}}$ After the event screenings, in ks.

within the area covered by the two analyzed Suzaku pointings. The other X-ray features detected with Suzaku at high significance level are missing any obvious Galactic or extragalactic identifications (NED or SIMBAD databases) and as such are considered below as "possibly related to the lobe"; these are marked in Figure 2 with white circles and denoted as "src 1-8." It should be noted that spurious X-ray features due to instrumental artifacts are present in the left-hand bottom corners of the two FOVs, and these are caused by the degradation of the CCD sensor XIS0 by the anomalous charge leakage. ${ }^{17}$

For the spectral analysis of the eight X-ray features classified as "possibly related to the lobe" we extracted the source fluxes from circular regions with radii 1.5 (well matched to the pointspread function (PSF) of the XIS instrument) centered on each spot (see Figure 2) and the background fluxes from larger ellipsoidal regions "BGD/Lobe 1" and "BGD/Lobe 2" chosen in the corresponding FOVs to avoid any compact feature. The

17 See the related 2010-01 memo at http://www.astro.isas.jaxa.jp/suzaku/doc/suzakumemo/. photon statistics for the analyzed regions are given in Table 2. Only src 3 and 5-8 are detected at a significance level above $5 \sigma$.

With the given photon statistics it is impossible to state if the "possibly related" spots are point-like for the XIS detector, or if they are (marginally) extended. It is also not possible at this point to state with high certainty if the detected X-ray features with no obvious counterparts at optical wavelengths are indeed physically associated with the giant Centaurus A structure. A comparison of the radio and X-ray morphologies presented in Section 2.3 indicates that this may indeed be the case, at least for some of them (see also the discussion in Section 3.2). And similarly, one cannot exclude the possibility of a chance coincidence between an X-ray spot associated with the giant lobe and a background/foreground optical object. Because the exact positions of the detected X-ray features cannot be measured given the angular resolution of the XIS instrument and the photon statistics that we obtain, no meaningful quantification of a chance probability for spurious association with a background/ foreground object can be made. Here we consider an association with such an object unlikely if all cataloged sources are 
Table 2

Photon Statistics for the Analyzed X-Ray Spots

\begin{tabular}{|c|c|c|c|}
\hline Region & (R.A., Decl. $^{\mathrm{a}}$ & Total Cnt. & $\begin{array}{l}\text { Net Cnt./ } \\
\text { Stat. Err. }\end{array}$ \\
\hline $\mathrm{BGD} / \mathrm{L} 1$ & & $1993 / 788^{c}$ & \\
\hline src 1 & $(200.949,-45.097)$ & 841 & $53 / 40$ \\
\hline src 2 & $(200.822,-45.116)$ & 890 & $102 / 41$ \\
\hline $\operatorname{src} 3$ & $(200.708,-45.177)$ & 1627 & $839 / 49$ \\
\hline $\operatorname{src} 4$ & $(200.851,-45.270)$ & $402^{\ddagger}$ & $4 / 28^{\ddagger}$ \\
\hline $\mathrm{BGD} / \mathrm{L} 2$ & $\ldots$ & $2370 / 781^{\mathrm{c}}$ & $\ldots$ \\
\hline $\operatorname{src} 5$ & $(200.377,-45.036)$ & 1081 & $300 / 43$ \\
\hline $\operatorname{src} 6$ & $(200.439,-45.113)$ & 1177 & $396 / 44$ \\
\hline src 7 & $(200.366,-45.198)$ & 1233 & $452 / 45$ \\
\hline $\operatorname{src} 8$ & $(200.300,-45.170)$ & 1468 & $687 / 47$ \\
\hline
\end{tabular}

Notes.

${ }^{a}$ Center positions for the circular regions (radii 1.5) used for the source flux extraction.

b Statistical errors calculated assuming Poisson distribution as a square root of a sum of the source and background XIS0+3 counts, except for src $4\left({ }^{\ddagger}\right)$, where only the XIS3 counts were considered (as the feature is located near the dead columns in XIS0).

${ }^{c}$ Counts normalized to the effective area of the circular source regions.

located more than 1.5 away from the centers of the circular regions used for the source flux extraction (see Table 2). For the selected features 1-8 analyzed further below, this criterion is marginally violated only in the case of src 1 , with the galaxy 2MASX J13234402-4505410 falling formally within the selected Suzaku source region. The analysis presented in Section 2.3 confirms, however, that the three prominent X-ray spots coinciding with bright optical stars, and as such classified as unrelated to the lobe, are spectrally distinct when compared with the other detected features. These other features are therefore not likely to be foreground stars, and instead we have to consider the possibility that they are associated with uncataloged background active galactic nuclei (AGNs).

In order to evaluate the expected number of background AGNs within the FOVs of the two Suzaku "on" pointings, we note that the $\log N-\log S$ distribution of such objects, constructed by Tozzi et al. (2001) based on the Chandra Deep Field-South observations and ROSAT observations of the Lockman Hole, gives the number of sources with $0.5-2 \mathrm{keV}$

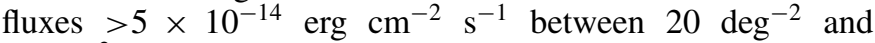
$3 \mathrm{deg}^{-2}$ (including the $1 \sigma$ uncertainties due to the sum of the Poisson noise). This implies a maximum of four background AGNs within the two effective FOVs of the XIS instrument $\left(2 \times 0.09 \mathrm{deg}^{-2}\right)$ at the accessible flux level, a factor of at least two below the detected number of the X-ray spots with no cataloged optical counterparts. The number of background X-ray sources evaluated by Tozzi et al. (2001) in the hard band, i.e., sources with $2-10 \mathrm{keV}$ fluxes $>5 \times 10^{-14} \mathrm{erg} \mathrm{cm}^{-2} \mathrm{~s}^{-1}$, is, on the other hand, higher, namely, $\sim 40 \mathrm{deg}^{-2}$ (as confirmed by other, more recent estimates, in agreement with the results of AGN population synthesis models; e.g., Ueda et al. 2003; Gandhi \& Fabian 2003; Georgakakis et al. 2008; Lehmer et al. 2012), meaning that we would expect about seven AGNs within the area of the giant lobe covered by the Suzaku observations. This number is close to the number of our detected X-ray spots with no obvious optical identification. Yet unidentified background AGNs are typically characterized by very flat spectra around keV photon energies (the average photon index $\Gamma=1.4$; Tozzi et al. 2001), while src 1-8 discussed here display on average much steeper X-ray continua (as we will show in Section 2.3). These spots therefore do not have the appearance of regular type 2 AGNs lacking optical identification due to a substantial intrinsic obscuration, with the possible exception of src 3. Moreover, we note that the two shorter "off" pointings with Suzaku reveal no bright X-ray features at the flux level of src 1-8 discussed here (which all lie well above the nominal limiting flux for the $20 \mathrm{ks}$ exposure time of the "off" pointings), except for objects with cataloged Galactic or extragalactic counterparts.

Hence, we conclude that while the contamination of the analyzed Suzaku pointings with unidentified background AGNs is an issue, there seems to be a slight overabundance of relatively bright and steep-spectrum X-ray sources in the "on" pointings when compared with both the "off" pointings and the expected $\log N-\log S$ distribution of background AGNs.

In order to elaborate more on the possible association of the detected X-ray spots with background AGNs, we have investigated the available mid-infrared (MIR) maps of the area covered by the Suzaku exposures provided by the NASA Widefield Infrared Survey Explorer satellite (WISE; Wright et al. 2010). Over the course of a year after its launch in late 2009, WISE carried out a deep all-sky survey in four MIR bands, $\mathrm{W} 1, \mathrm{~W} 2, \mathrm{~W} 3$, and $\mathrm{W} 4$, centered on wavelengths around 3.4, $4.6,12$, and $22 \mu \mathrm{m}$, respectively. The PSF of the telescope corresponds to a Gaussian of about $6^{\prime \prime}, 6^{\prime \prime}, 6^{\prime \prime}$, and $12^{\prime \prime}$ in W1-W4, respectively, sampled at 2.8, 2.8, 2.8, and 5.'6 pixel ${ }^{-1}$. With nominal $5 \sigma$ point-source sensitivities of $\sim 0.08,0.1,1$, and $6 \mathrm{mJy}$ in the four bands, this survey is orders of magnitude more sensitive than previous infrared all-sky surveys (Soifer et al. 1987; Ishihara et al. 2010; Yamamura et al. 2010). The WISE pipeline implements a sophisticated multi-band and multi-frame source detection algorithm. The final product is an all-sky survey data release catalog ${ }^{18}$ that reports Vega source magnitudes with the absolute calibration being referenced to standard stars.

The corresponding images in the short (W1) and long (W4) wavelength bands are shown in Figure 3. The all-sky data release was searched for detected objects within a radius of 1.5 around the positions of the region centers listed in Table 2. All sources with the photometric quality (ph_qual) flag of $\mathrm{A}, \mathrm{B}$, or $\mathrm{C}$ denoting real (albeit including low signal to noise) detections were included. The selection was carried out for each band individually. We used profile-fit magnitudes and standard zero points (Jarrett et al. 2011) in order to measure the approximate contribution due to associated point sources. The summed fluxes of detections for each region are listed in Table 3. These should be treated as upper limits on the contribution of point sources at the position of each X-ray feature discussed here.

Figure 3 shows that the areal density of WISE detections in the targeted part of the sky decreases strongly with wavelength, with each source extraction region for all the analyzed X-ray spots overlapping with several MIR emitters at $3.4 \mu \mathrm{m}$, but being typically devoid of any bright sources at $22 \mu \mathrm{m}$. Only the regions of src 1 and 3 are completely dominated by a couple of sources alone. In particular, src 1 coincides with one very blue object at $($ R.A. $=200.9314$, decl. $=-45.0827$ ), a star, TYC 8248-535-1, present in the SIMBAD database, and one red object at $(200.9335,-45.0947)$, the aforementioned galaxy 2MASX J13234402-4505410. The brightest MIR emitter in the region of src 3 is a very red source located at (200.7099, $-45.1809)$, lacking any optical identification.

\footnotetext{
18 http://wise2.ipac.caltech.edu/docs/release/allsky
} 

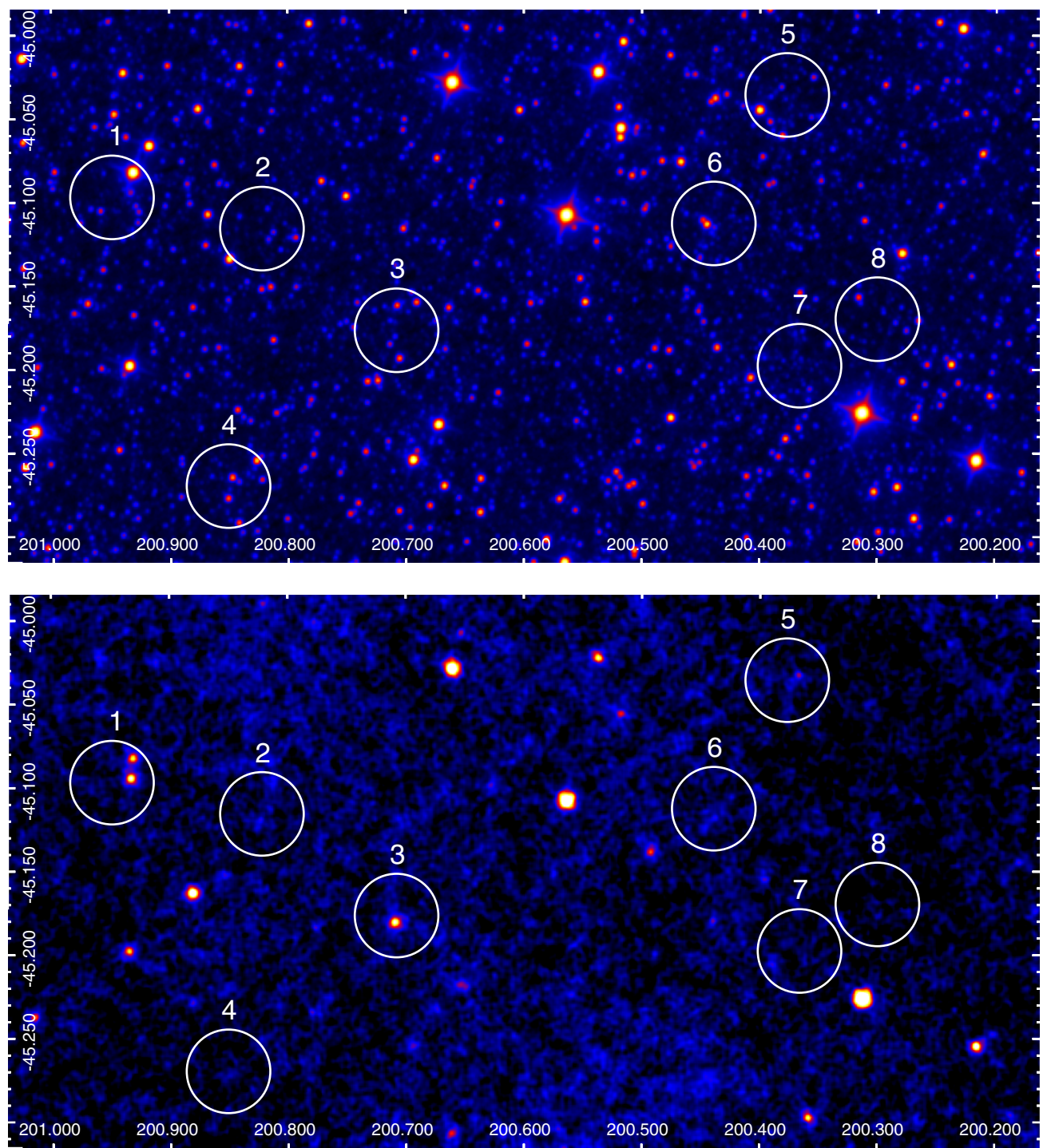

Figure 3. WISE images of the part of the sky targeted by the two Suzaku "on" pointings at short (W1) and long (W4) mid-infrared wavelength (upper and lower panels, respectively). White circles denote the source extraction regions for the analyzed X-ray spots, as given in Table 2 and shown in Figure 2.

(A color version of this figure is available in the online journal.)

Table 3

Total MIR Fluxes (in mJy) for the Source Extraction Regions of the X-Ray Spots

\begin{tabular}{|c|c|c|c|c|c|c|}
\hline Region & $\mathrm{W} 1(3.4 \mu \mathrm{m})$ & $\mathrm{W} 2(4.6 \mu \mathrm{m})$ & W3 $(12 \mu \mathrm{m})$ & $\mathrm{W} 4^{\mathrm{a}}(22 \mu \mathrm{m})$ & $\alpha_{3.4 \mu \mathrm{m}-1 \mathrm{keV}}$ & $\alpha_{22 \mu \mathrm{m}-1 \mathrm{keV}}$ \\
\hline $\operatorname{src} 1^{\mathrm{b}}$ & 287.8 & 141.0 & 34.2 & 17.7 & 2.2 & 1.5 \\
\hline $\operatorname{src} 2$ & 10.9 & 5.9 & 3.7 & $<6$ & 1.8 & $<1.4$ \\
\hline $\operatorname{src} 3^{c}$ & 33.7 & 18.1 & 10.7 & 14.8 & 1.8 & 1.4 \\
\hline $\operatorname{src} 4$ & 49.1 & 25.3 & 4.8 & $<6$ & 2.0 & $<1.4$ \\
\hline $\operatorname{src} 5$ & 58.6 & 30.2 & 7.1 & 8.6 & 2.0 & 1.4 \\
\hline $\operatorname{src} 6$ & 35.1 & 17.8 & 7.0 & $<6$ & 1.9 & $<1.4$ \\
\hline $\operatorname{src} 7$ & 11.4 & 6.2 & 3.1 & $<6$ & 1.7 & $<1.3$ \\
\hline $\operatorname{src} 8$ & 17.6 & 9.4 & 2.1 & $<6$ & 1.8 & $<1.3$ \\
\hline
\end{tabular}

Notes.

${ }^{a}$ For the W4 filter, several regions are devoid of detections; in these cases, the nominal $5 \sigma$ point-source detection limit of $6 \mathrm{mJy}$ has been assumed.

${ }^{\mathrm{b}}$ The total WISE flux is dominated at short wavelengths by a star TYC 8248-535-1, and at longer wavelengths by a galaxy 2MASX J13234402-4505410.

c The total WISE flux is dominated by a single red object located at $\left(200^{\circ} 7099,-45^{\circ} 1809\right)$ lacking any optical identification. 
Table 4

Modeling Results for the X-Ray Spots Detected at $>5 \sigma$ Level

\begin{tabular}{|c|c|c|c|c|c|c|c|c|c|c|c|}
\hline $\begin{array}{l}\text { Source } \\
\text { (1) }\end{array}$ & $\begin{array}{c}\text { Red. } \chi^{2} / \text { dof } \\
\text { (2) }\end{array}$ & $\begin{array}{c}\Gamma \\
(3)\end{array}$ & $\begin{array}{c}F_{\mathrm{X}, \mathrm{abs}} \\
(4)\end{array}$ & $\begin{array}{l}F_{\mathrm{X}} \\
(5)\end{array}$ & $\begin{array}{c}\text { Red. } \chi^{2} / \text { dof } \\
(6)\end{array}$ & $\begin{array}{l}k T \\
(7)\end{array}$ & $\begin{array}{c}F_{\mathrm{X}, \mathrm{abs}} \\
(8)\end{array}$ & $\begin{array}{l}F_{\mathrm{X}} \\
(9)\end{array}$ & $\begin{array}{c}Z / Z_{\odot} \\
(10)\end{array}$ & $\begin{array}{l}\text { Norm } \\
(11)\end{array}$ & $\begin{array}{c}\text { Red. } \chi^{2} / \text { dof } \\
\text { (12) }\end{array}$ \\
\hline $\operatorname{src} 3$ & $1.340 / 8$ & $1.32_{-0.12}^{+0.12}$ & 29.2 & 30.4 & $0.671 / 51$ & 32.8 & 28.3 & 29.5 & $0.3^{f}$ & $17.1_{-2.2}^{+3.6}$ & $0.639 / 52$ \\
\hline $\operatorname{src} 5$ & $0.211 / 8$ & $2.00_{-0.32}^{+0.35}$ & 6.6 & 7.3 & $1.192 / 30$ & $4.6_{-1.6}^{+4.7}$ & 6.3 & 6.8 & $0.3^{f}$ & $4.77_{-0.82}^{+0.81}$ & $1.249 / 31$ \\
\hline $\operatorname{src} 6$ & $0.395 / 8$ & $1.89_{-0.21}^{+0.24}$ & 7.5 & 8.3 & $0.804 / 36$ & $4.6_{-1.3}^{+2.6}$ & 6.9 & 7.4 & $0.3^{f}$ & $5.24_{-0.67}^{+0.66}$ & $0.788 / 37$ \\
\hline $\operatorname{src} 7$ & $0.693 / 8$ & $2.52_{-0.23}^{+0.24}$ & 7.1 & 8.3 & $1.020 / 38$ & $2.1_{-0.3}^{+0.7}$ & 5.8 & 6.5 & $0.3^{f}$ & $6.55_{-0.69}^{+0.69}$ & $1.373 / 39$ \\
\hline $\operatorname{src} 8$ & $0.598 / 8$ & $1.68_{-0.14}^{+0.14}$ & 15.8 & 16.8 & $0.685 / 47$ & $7.3_{-2.1}^{+4.3}$ & 14.9 & 15.8 & $0.3^{f}$ & $9.57_{-0.71}^{+0.73}$ & $0.770 / 48$ \\
\hline
\end{tabular}

Notes. (1) Source ID; (2) quality of a constant fit to the source light curve (reduced $\chi^{2}$ value/degree of freedom); (3) photon index in the PL model with $90 \%$ confidence level errors; (4) absorbed $0.5-10 \mathrm{keV}$ flux of the source in the PL model, in units of $10^{-14} \mathrm{erg} \mathrm{cm}^{-2} \mathrm{~s}^{-1} ;(5)$ unabsorbed $0.5-10 \mathrm{keV}$ flux of the source in the PL model in the same units; (6) quality of the PL fit; (7) plasma temperature for the APEC model in units of keV with $90 \%$ confidence level errors (except for src 3, for which no meaningful errors could be calculated); (8) absorbed 0.5-10 keV flux of the source in the APEC model, in units of $10^{-14} \mathrm{erg} \mathrm{cm}^{-2} \mathrm{~s}^{-1}$; (9) unabsorbed $0.5-10 \mathrm{keV}$ flux of the source in the APEC model in the same units; (10) fixed (" $f$ ") abundance in the APEC fit; (11) APEC normalization $\times 10^{-5}$; (12) quality of the APEC fit.

The last two columns in Table 3 list the corresponding MIRto-X-ray spectral indices, computed formally with the given $3.4 \mu \mathrm{m}$ and $22 \mu \mathrm{m}$ fluxes, and $1 \mathrm{keV}$ fluxes of each spot (see Section 2.3). The provided numbers do not correspond necessarily to real source spectra, as the utilized WISE fluxes are superpositions of contributions from distinct objects, especially at shorter wavelengths. We note, however, that the evaluated $\alpha_{3.4 \mu \mathrm{m}-1 \mathrm{keV}}$ indices are relatively steep, $\gtrsim 1.7$, so even assuming that the W1-band fluxes of real counterparts of the X-ray features are one order of magnitude lower than the listed values, these indices would still all be $>1$. The situation is somewhat different

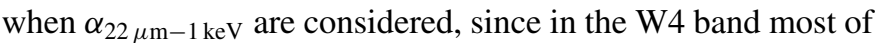
the analyzed regions (including X-ray bright src 6-8) are lacking significant detections. These non-detections may potentially challenge the possibility for AGN associations, as the MIR-to$\mathrm{X}$-ray power-law slopes for local radio-quiet AGNs have been shown to be $\gtrsim 1$ (e.g., Gandhi et al. 2009).

All in all, based on the analysis of the WISE data, we conclude that at least src 3-clearly coinciding with a red MIR emitter-is consistent with being a background obscured AGN rather than a feature related to the lobe. This conclusion is supported by the X-ray spectral analysis presented in the next section. In addition, the relatively dim src 1 could be considered as a likely X-ray counterpart of a "low-luminosity AGN." As for the other $\mathrm{X}$-ray spots considered, the situation is still open, especially as more AGN associations could be expected on statistical grounds. We draw attention to the particularly interesting cases of src 7 and 8, which lack any optical or MIR counterparts but at the same time, as shown below, are characterized by relatively steep $\mathrm{X}$-ray spectra and positionally associated with a prominent radio filament in the lobe.

\subsection{X-Ray Spots: Spectral Analysis}

We fitted the spectra of the X-ray features detected at significance levels above $5 \sigma$ (src 3 and 5-8) using XSPEC (ver. 12.7.0) with an absorbed power-law model (PL) and an absorbed thermal model APEC (Foster et al. 2012), in all cases freezing the absorbing neutral hydrogen column density at the Galactic value in the direction of the source, $N_{\mathrm{H}, \mathrm{Gal}}=0.7 \times 10^{21} \mathrm{~cm}^{-2}$. The results of the fitting, summarized in Table 4, indicate that both models provide an equally good description of the data, with the emerging photon indices $\Gamma \simeq 2.0 \pm 0.5$ for all the spots, except for src 3 , which is characterized by $\Gamma<1.5$, or equivalently with plasma temperatures within the range $k T \sim 1-10 \mathrm{keV}$, again except for src 3 , which has a poorly constrained $k T>10 \mathrm{keV}$.
Figure 4 presents the Suzaku spectra of src 3 and 5-8 and the corresponding PL fits. The summed unabsorbed $0.5-10 \mathrm{keV}$ flux of all the analyzed features is $F_{\mathrm{X}} \simeq 7 \times 10^{-13} \mathrm{erg} \mathrm{cm}^{-2} \mathrm{~s}^{-1}$, implying a total $0.5-10 \mathrm{keV}$ luminosity of the spots $L_{\mathrm{X}} \sim$ $10^{39} \mathrm{erg} \mathrm{s}^{-1}$ (for the assumed distance of $D=3.7 \mathrm{Mpc}$ ). These values correspond to a surface area $2 \times 0.09 \mathrm{deg}^{2}$, i.e., twice the effective FOV of the XIS instrument. All the analyzed $\mathrm{X}$-ray sources appear steady during the performed exposures, as shown in Figure 5 and quantified in Table 4. We note that in the fitting procedure using the APEC model, due to the rather limited photon statistics, the plasma abundance could not be treated as a free parameter but instead was fixed at the value $Z=0.3 Z_{\odot}$ (as roughly expected for the intergalactic medium at larger distances from the galaxy group/cluster center; see, e.g., Sun 2012). Alternative fits with the abundance reduced to $Z=0.1 Z_{\odot}$, or even assuming a pure bremsstrahlung model instead of APEC, returned essentially the same model parameters (including normalization) and comparable $\chi^{2}$ values.

As follows from Table 4, src 3 stands out in terms of its flux and spectral properties from the other X-ray spots analyzed here, displaying a flat X-ray spectrum with no signatures for curvature or a cutoff up to $10 \mathrm{keV}$ photon energies. This justifies further the idea, presented previously in Section 2.2 based on the analysis of the WISE data, that this feature is related to a background type 2 AGN rather than to the Centaurus A giant lobe. ${ }^{19}$ With src 3 excluded, the summed $0.5-10 \mathrm{keV}$ fluxes and luminosities of the modeled src 5-8 are $F_{\mathrm{X}} \simeq 4 \times 10^{-13} \mathrm{erg} \mathrm{cm}^{-2} \mathrm{~s}^{-1}$ and $L_{\mathrm{X}} \sim 0.6 \times 10^{39} \mathrm{erg} \mathrm{s}^{-1}$, respectively, meaning $F_{\mathrm{X}} \sim$ $10^{-13} \mathrm{erg} \mathrm{cm}^{-2} \mathrm{~s}^{-1}$ and $L_{\mathrm{x}} \sim 10^{38} \mathrm{erg} \mathrm{s}^{-1}$ per spot, on average.

Figure 6 presents the superposition of the X-ray contours on the polarized radio intensity map of the southern lobe in Centaurus A. As shown, some of the bright X-ray features classified before as "possibly related to the lobe" do indeed coincide with the polarized radio structures. The correlation is particularly evident in the case of the bright spots 7 and 8, located almost exactly at the position of the two "holes" within a single bright radio filament. This kind of morphology reminds us of the structures observed around the Galactic center region, consisting of highly polarized radio filaments (Lang et al. 1999; LaRosa et al. 2000; Nord et al. 2004; Yusef-Zadeh et al. 2004) interacting with molecular clouds (Staguhn et al. 1998) and coinciding in

\footnotetext{
19 We note some hints for the presence of a line-like feature/excess in the Suzaku spectrum of src 3 (both front- and back-illuminated CCDs), around the observed photon energies of $\simeq 4 \mathrm{keV}$. If due to iron $\mathrm{K} \alpha$ emission, this feature would be consistent with a source redshift of, roughly, $z \sim 0.5$.
} 

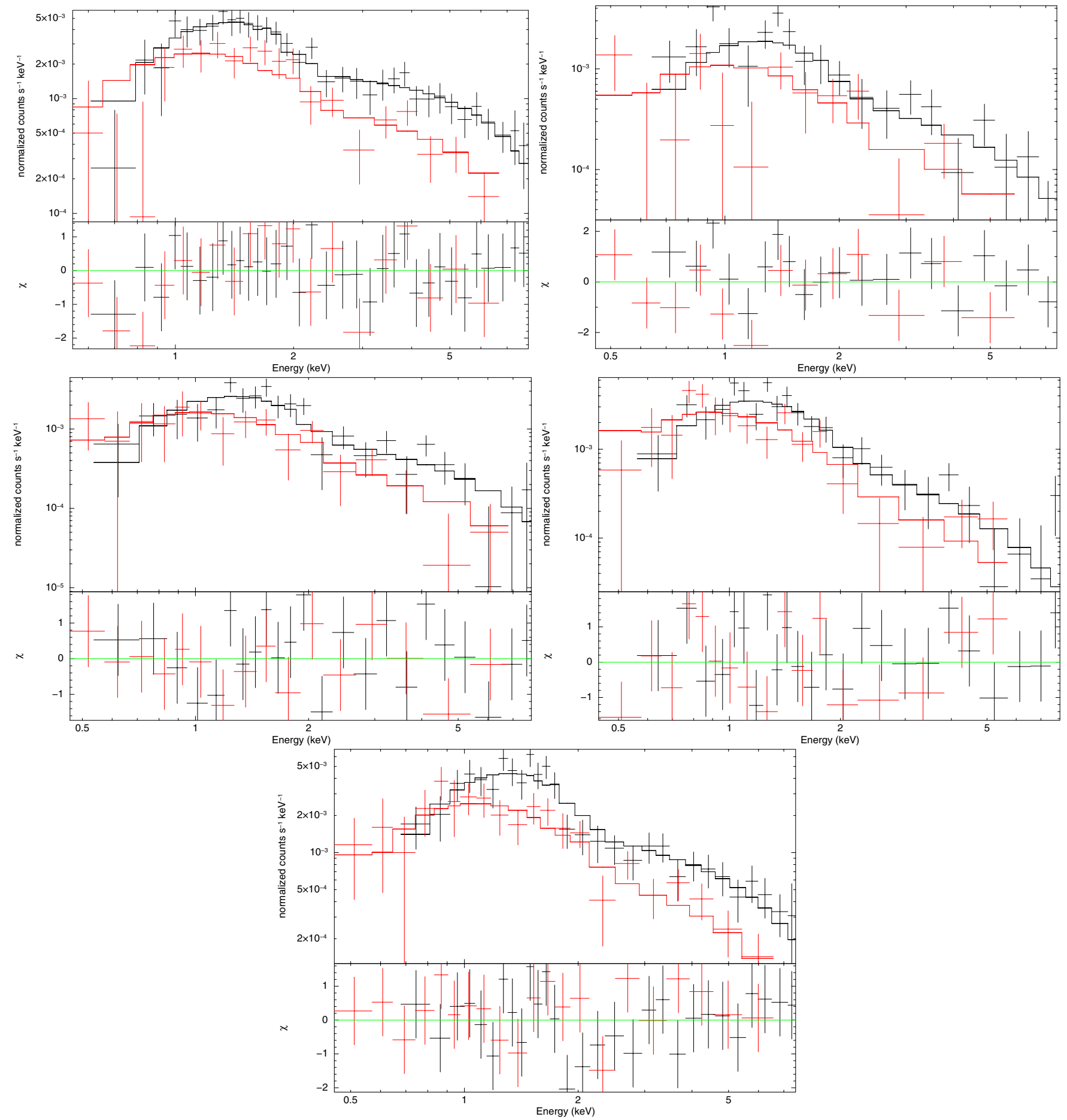

Figure 4. X-ray spectra of sources 3 and 5-8 (from top to bottom, respectively), as measured by front-illuminated CCDs XIS0+3 (black data points) and back-illuminated CCD XIS1 (red data points). Continua represent PL models fitted to the data (Table 4 and Section 2.3). The data points are binned in 40 counts.

(A color version of this figure is available in the online journal.)

many cases with compact X-ray features of unknown origin (Sakano et al. 2003; Lu et al. 2008; Johnson et al. 2009; see also Section 3).

If the two "on" regions targeted with Suzaku are representative of the rest of the halo, one may estimate the expected total $0.5-10 \mathrm{keV}$ flux and the total $0.5-10 \mathrm{keV} \mathrm{lu}-$ minosity of the entire population of the X-ray spots "possibly related to the radio structure" (with src 3 excluded) to be $F_{\mathrm{X}, \text { tot }} \sim F_{\mathrm{X}} \times 13.2 \mathrm{deg}^{2} / 0.18 \mathrm{deg}^{2} \sim 3 \times 10^{-11} \mathrm{erg} \mathrm{cm}^{-2} \mathrm{~s}^{-1}$ and $L_{\mathrm{X}, \text { tot }} \simeq 4 \pi D^{2} F_{\mathrm{X} \text {, tot }} \sim 5 \times 10^{40} \mathrm{erg} \mathrm{s}^{-1}$, respectively, where $13.2 \mathrm{deg}^{2}$ is the total angular extension of the Centaurus A giant lobes on the sky (following Hardcastle et al. 2009). These are non-negligible values indeed, taking into account that the single previously available SAS3 upper limit for the X-ray emission of the halo is of the same order (Marshall \& Clark 1981). Even more importantly, the total expected non-thermal (IC) flux of the giant lobes within the $0.5-10 \mathrm{keV}$ range, as derived from the broadband modeling presented in Abdo et al. 

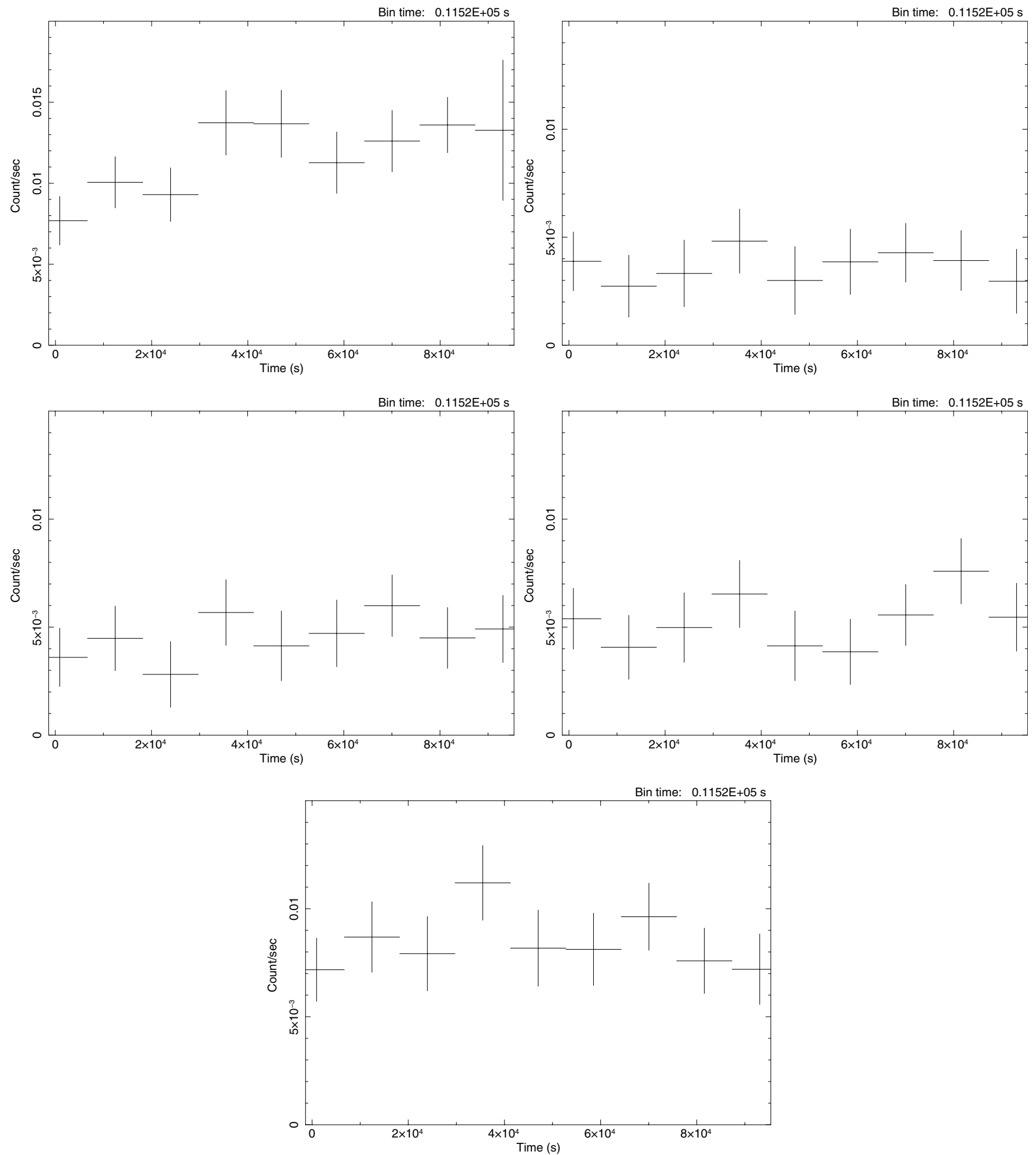

Figure 5. X-ray light curves of sources 3 and 5-8 (from top to bottom, respectively) during the corresponding Suzaku exposures, binned in $3.2 \mathrm{hr}$ intervals. The performed constant fits to the light curves imply steady emission during the performed exposures (see Table 4 and Section 2.3).

(2010), is $\simeq 1.2 \times 10^{-11} \mathrm{erg} \mathrm{cm}^{-2} \mathrm{~s}^{-1}$, which is formally below the expected summed contribution from the spots. We also note that the total flux of the diffuse thermal emission detected in our Suzaku observations within the Centaurus A lobes is similarly of the same order of magnitude (see Section 2.4 below). It should be kept in mind, however, that even if the discussed spots are indeed related to the lobes, their distribution within the whole giant halo may not be uniform, as the two analyzed Suzaku pointings-which targeted small but particularly radio-bright parts of the southern lobe-may not be representative of the rest of the extended structure. Regardless, one may conclude that the total X-ray emission of giant lobes in systems like Centaurus A is a complex mixture of the diffuse thermal and non-thermal components, plus a prominent contribution from compact 


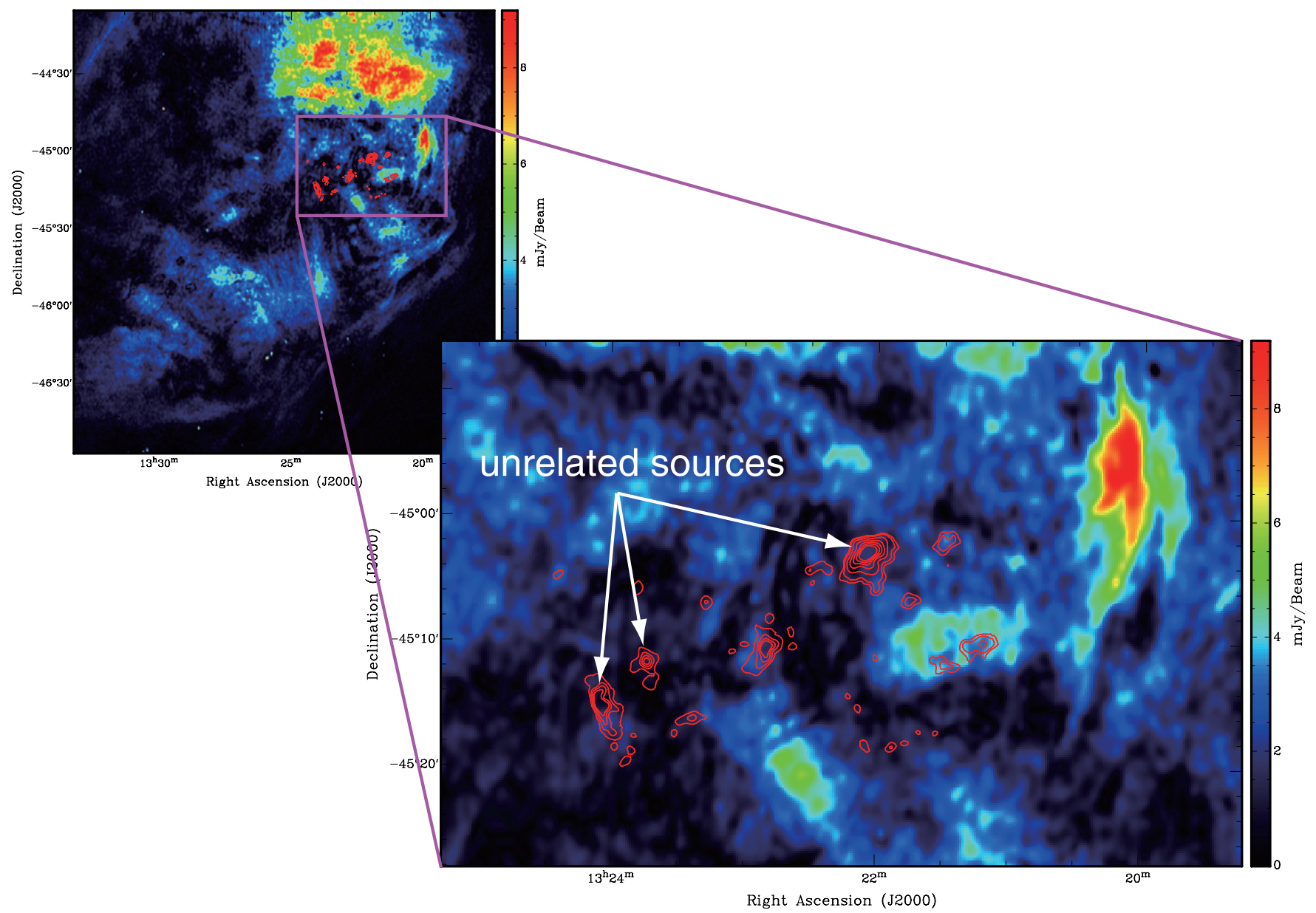

Figure 6. Polarized radio intensity map of the Centaurus A southern lobe with the X-ray Suzaku contours superimposed. Note that the most pronounced radio filament in the top-right corner of the zoomed image was unfortunately not covered by the Suzaku observations.

X-ray features. Only with the broad energy coverage and suitable combination of sensitivity and resolution of Suzaku could these components be disentangled, as otherwise they would form a single emission continuum.

Finally, let us comment on the three X-ray spots coinciding with bright stars and as such classified before as unrelated to the lobes. Their XIS spectra, extracted in the same way as in the cases of src 3 and 5-8 discussed above, did not give good fits with the PL model (with $N_{\mathrm{H}}$ set free), or the absorbed APEC model assuming an abundance of 0.3 solar or higher. Only with the abundance in the APEC model set free and zero absorption could reasonable fits be obtained, returning $Z<Z_{\odot}$ and $k T \lesssim 1 \mathrm{keV}$. Low abundance in fits of this kind has been shown often to be an artifact resulting from fitting to a mixedtemperature plasma (see the related discussion in Kim 2012). And, indeed, the X-ray brightest spot coinciding with stars HD 116099/TYC 8248-981-1 could formally be fitted well with a two-component APEC model. As noted above, this difference in the X-ray spectrum supports our belief that these sources are unrelated to the lobes.

\subsection{Diffuse Emission Component}

In the previous sections we focused on compact X-ray features detected in the Suzaku "on" pointings "Lobe 1" and "Lobe 2" centered on the southern lobe in the Centaurus A radio galaxy. In this section we present a detailed analysis of the diffuse emission component filling the whole FOV of the XIS instrument, which remains after removing all the compact $X$-ray features, including src 1-8, three spots coinciding with bright optical stars, and spurious sources due to instrumental artifacts. The diffuse counts extracted from the entire FOV of both CCDs were analyzed using standard methods, utilizing the energy range $0.6-7.0 \mathrm{keV}$ for XIS0 and XIS3 and $0.5-6.0 \mathrm{keV}$ for XIS1 (to avoid large errors in the extrapolation of contamination files), and ignoring the range $1.8-1.9 \mathrm{keV}$ due to calibration uncertainty of Si K-edge. The ARF files were produced assuming that the diffuse emission is distributed uniformly within circular regions with $20^{\prime}$ radii (giving the ARF area of $0.35 \mathrm{deg}^{2}$ ) using xissimarfgen and new contamination files. ${ }^{20}$ The background component was estimated from the two shorter "off" Suzaku exposures "Lobe 3" and "Lobe 4" targeting the blank sky outside of (but close to) the Centaurus A southern lobe (see the last two rows of Table 1), again after removing all the prominent compact features.

Figure 7 presents the large-scale and broadband ROSAT count map centered on the position of Centaurus A, together with the $4.75 \mathrm{GHz}$ contour map of the giant lobes (Junkes et al. 1993); the positions of all the Suzaku pointings (see Table 1) are marked with yellow squares. The XIS0+3 images of the "off" pointings, corresponding to the photon energy range $0.5-10 \mathrm{keV}$, are shown in Figure 8, with optical sources from the SIMBAD database denoted by green crosses. In the figure, white circles and ellipses

\footnotetext{
${ }^{20}$ Contamination files ae_xi?_contami_edm_20110927.fits taken from http://space.mit.edu/XIS/monitor/contam/.
} 


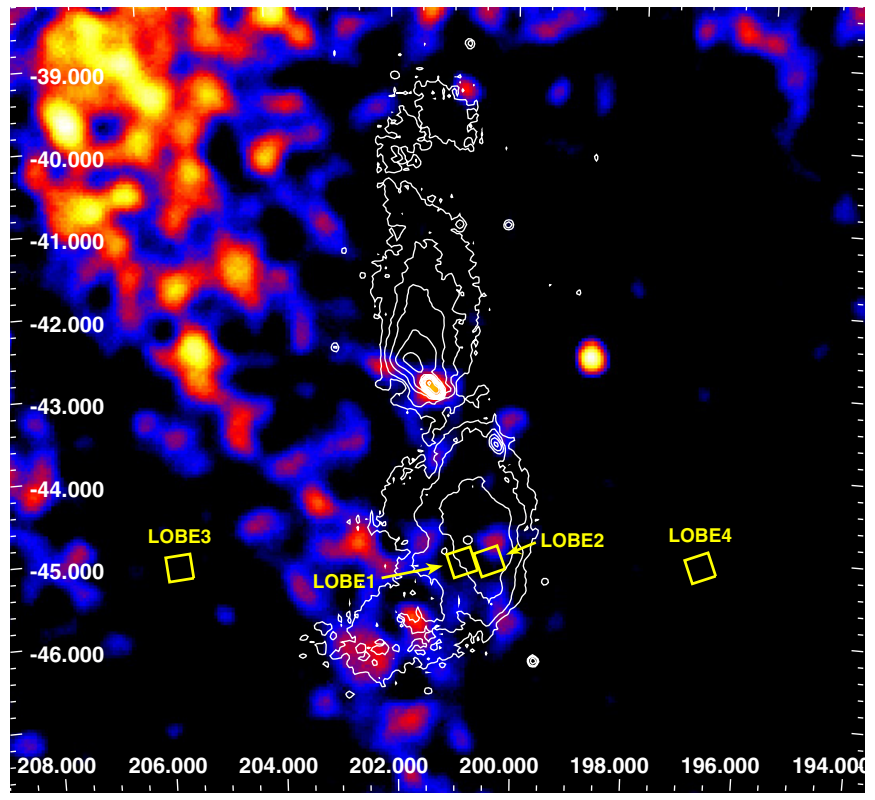

Figure 7. Broadband ROSAT count map smoothed with a Gaussian with $\sigma=$ 7 pixels and centered on the position of the Centaurus A radio galaxy. The white contours correspond to the $4.75 \mathrm{GHz}$ Parkes image of the giant structure (Junkes et al. 1993), beginning at $0.1 \mathrm{Jy} \mathrm{beam}^{-1}$ and increasing by a factor of two. The positions of all the Suzaku pointings analyzed in this paper (see Table 1) are represented with yellow squares and labeled with their observation number.

(A color version of this figure is available in the online journal.)

mark the positions of compact X-ray features (astrophysical sources or instrument artifacts in the bottom-left corners of the two FOVs) that are removed from the extraction areas used to measure spectra for the diffuse counts. We note that the most prominent X-ray feature in the "Lobe 4" region (lower panel in Figure 8), with $0.5-10 \mathrm{keV}$ flux comparable to those of src $1-8$ analyzed previously, coincides with a strong $(\sim 10 \mathrm{mJy})$ radio source SUMSS J130713-451427.

First, we fit all four Suzaku pointings using XSPEC with the same model MEKAL + wabs $*($ MEKAL + PL) including an unabsorbed thermal component representing Local Bubble (LB) emission, an absorbed thermal component representing Galactic halo (GH) emission, and an absorbed power-law component corresponding to the cosmic X-ray background (CXB) radiation (see, e.g., Yuasa et al. 2009). The neutral hydrogen column density was fixed as before to the Galactic value in the direction of the source, $N_{\mathrm{H}, \mathrm{Gal}}=0.7 \times 10^{21} \mathrm{~cm}^{-2}$, the photon index for the CXB component was fixed at $\Gamma_{\mathrm{CXB}}=1.41$, and the abundance in the MEKAL models was fixed at the solar value. The results of the fitting are summarized in Table 5. As shown, while the $\mathrm{LB}$ and $\mathrm{CXB}$ components are reproduced correctly in all four cases, an excess emission in the "on" pointings seems to be present when compared with both "off" pointings, manifesting itself in the increased (by a factor of two) normalization of the GH component in the "Lobe 1" and "Lobe 2" regions. This excess may represent a largescale fluctuation in the $\mathrm{GH}$ emission, or an additional X-ray component originating in the Centaurus A system. The same result is obtained using the APEC plasma model instead of MEKAL, along with an alternative parameterization of the Galactic foreground and a complementary fitting procedure, as summarized in the Appendix.

Next, we fit the "Lobe 1" and "Lobe 2" regions assuming the model MEKAL + wabs $*($ MEKAL $+\mathrm{PL}+\mathrm{MEKAL})$, with the
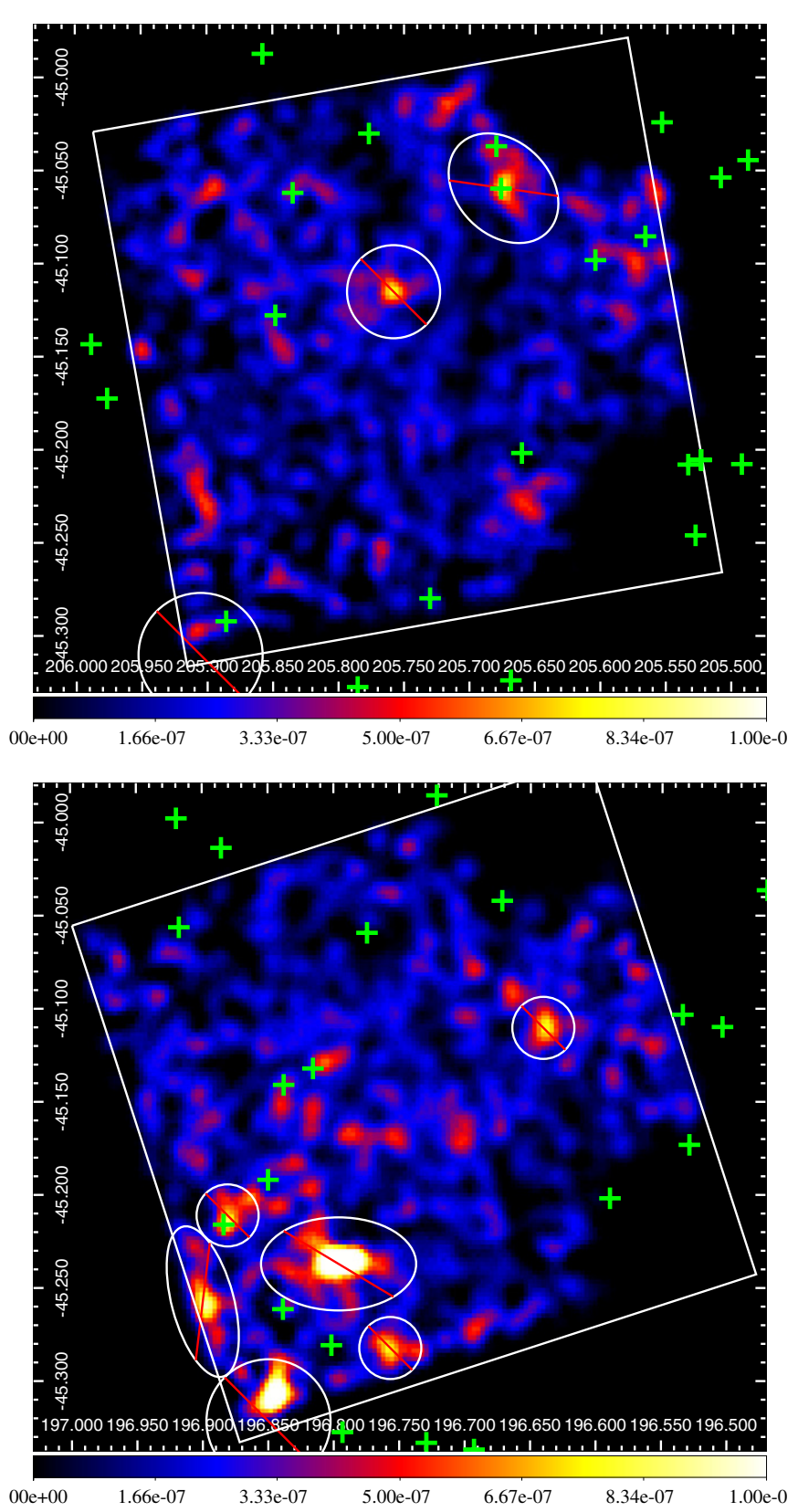

Figure 8. Vignetting-corrected and NXB-subtracted XISO+3 image of the "off" pointings ("Lobe 3" and "Lobe 4" in the upper and lower panels, respectively; see Table 1), with optical sources from the SIMBAD database denoted by green crosses. White circles and ellipses mark the positions of compact X-ray features that are removed from the extraction area used for the diffuse counts.

(A color version of this figure is available in the online journal.)

parameters (temperatures and normalizations) of the $\mathrm{LB}+$ $\mathrm{GH}+\mathrm{CXB}$ background emission fixed as derived previously based on the analysis of the "Lobe 3" region (see Table 5) and an additional absorbed thermal component representing the soft excess seen at the position of the lobes. The results are summarized in Table 6. The same procedure using "Lobe 4" instead of "Lobe 3" background parameters resulted in a rather poor description of the data (large values of reduced $\chi^{2}$ ), because of a larger number of point sources present in the "Lobe 4" pointing that had to be removed before the extraction of the diffuse counts, leading to a poorer background determination. We repeated the fits assuming an abundance for the additional thermal component of $Z / Z_{\odot}=0.1,0.3$, and 1 . As expected, the 
Table 5

Modeling Results for the Diffuse Emission

\begin{tabular}{|c|c|c|c|c|c|c|c|c|c|}
\hline $\begin{array}{l}\text { Region } \\
\text { (1) }\end{array}$ & $\begin{array}{c}k T_{\mathrm{LB}} \\
(2)\end{array}$ & $\begin{array}{c}\operatorname{Norm}_{L B} \\
\text { (3) }\end{array}$ & $\begin{array}{c}k T_{\mathrm{GH}} \\
(4)\end{array}$ & $\begin{array}{c}\operatorname{Norm}_{G H} \\
(5)\end{array}$ & $\begin{array}{c}\text { Norm }_{C X B} \\
(6)\end{array}$ & $\begin{array}{c}F_{0.5-2 \mathrm{keV}}^{\mathrm{abs}} \\
(7)\end{array}$ & $\begin{array}{c}F_{2-10 \mathrm{keV}}^{\mathrm{abs}} \\
(8)\end{array}$ & $\begin{array}{c}Z / Z_{\odot} \\
(9)\end{array}$ & $\begin{array}{c}\text { Red. } \chi^{2} / \text { dof } \\
(10)\end{array}$ \\
\hline Lobe 1 (“on”) & $0.19 \pm 0.01$ & $3.53_{-0.20}^{+0.20}$ & $0.60_{-0.03}^{+0.03}$ & $7.42_{-0.82}^{+0.79}$ & $0.97_{-0.02}^{+0.03}$ & 7.88 & 6.32 & $1.0^{f}$ & $1.16 / 441$ \\
\hline Lobe 2 (“on”) & $0.20 \pm 0.01$ & $3.22_{-0.17}^{+0.18}$ & $0.66_{-0.03}^{+0.02}$ & $7.38_{-0.69}^{+0.68}$ & $1.01_{-0.02}^{+0.03}$ & 7.67 & 6.59 & $1.0^{f}$ & $1.13 / 512$ \\
\hline Lobe 3 (“off”) & $0.20 \pm 0.01$ & $3.30_{-0.32}^{+0.33}$ & $0.70_{-0.09}^{+0.12}$ & $3.79_{-1.16}^{+1.28}$ & $1.04_{-0.05}^{+0.05}$ & 7.19 & 6.73 & $1.0^{f}$ & $1.30 / 131$ \\
\hline Lobe 4 ("off") & $0.19 \pm 0.01$ & $2.34_{-0.31}^{+0.33}$ & $0.80_{-0.13}^{+0.16}$ & $3.45_{-0.84}^{+0.88}$ & $1.18_{-0.06}^{+0.05}$ & 5.87 & 7.63 & $1.0^{f}$ & $0.87 / 123$ \\
\hline
\end{tabular}

Notes. (1) Region fitted; (2) temperature of the LB component in keV units; (3) MEKAL normalization of the LB component $\times 10^{-3}$; (4) temperature of the GH component in keV units; (5) MEKAL normalization of the GH component $\times 10^{-4}$; (6) normalization of the $\mathrm{CXB}$ component $\times 10^{-3}$; (7) absorbed $0.5-2 \mathrm{keV}$ flux in units of $10^{-12} \mathrm{erg} \mathrm{cm}^{-2} \mathrm{~s}^{-1} / 0.35 \mathrm{deg}^{2}$; (8) absorbed 2-10 keV flux in units of $10^{-12} \mathrm{erg} \mathrm{cm}^{-2} \mathrm{~s}^{-1} / 0.35 \mathrm{deg}^{2}$; (9) fixed (" $f$ ') abundance; (10) reduced $\chi^{2}$ value/degree of freedom.

Table 6

Modeling Results for the Excess Diffuse Emission

\begin{tabular}{lcccc}
\hline \hline$Z / Z_{\odot}$ & $\begin{array}{c}k T \\
(1)\end{array}$ & $\begin{array}{c}\text { Norm } \\
(2)\end{array}$ & $\begin{array}{c}F_{0.5-2 \mathrm{keV}}^{\mathrm{abs}} \\
(4)\end{array}$ & $\begin{array}{c}\text { Red. } \chi^{2} / \mathrm{dof} \\
(5)\end{array}$ \\
\hline $\begin{array}{lccc}\text { Lobe } 1 \\
1.0^{f}\end{array}$ & $0.46_{-0.11}^{+0.08}$ & $3.11_{-0.53}^{+1.11}$ & 5.84 & $1.20 / 444$ \\
$0.3^{f}$ & $0.45_{-0.10}^{+0.08}$ & $9.76_{-1.88}^{+3.80}$ & 6.12 & $1.20 / 444$ \\
$0.1^{f}$ & $0.39_{-0.07}^{+0.09}$ & $27.3_{-7.2}^{+10.4}$ & 6.72 & $1.20 / 444$ \\
\hline Lobe 2 & & & & \\
$1.0^{f}$ & $0.64_{-0.05}^{+0.05}$ & $2.91_{-0.35}^{+0.34}$ & 5.83 & $1.13 / 515$ \\
$0.3^{f}$ & $0.64_{-0.05}^{+0.05}$ & $8.59_{-1.03}^{+1.03}$ & 6.20 & $1.13 / 515$ \\
$0.1^{f}$ & $0.62_{-0.06}^{+0.06}$ & $19.1_{-2.49}^{+2.61}$ & 6.72 & $1.13 / 515$ \\
\hline
\end{tabular}

Notes. (1) The assumed abundance $Z$; (2) temperature of the additional thermal component in keV units; (3) the MEKAL normalization of the additional thermal component $\times 10^{-4}$; (4) absorbed $0.5-2 \mathrm{keV}$ flux in units of $10^{-13} \mathrm{erg} \mathrm{cm}^{-2} \mathrm{~s}^{-1} /$ $0.35 \mathrm{deg}^{2} ;(5)$ reduced $\chi^{2}$ value/degree of freedom.

derived model parameters are not sensitive to the particular value of the metallicity assumed, except for normalization, which roughly anti-correlates with $Z$. We note that in the case where the soft excess is related to a large-scale fluctuation of the $\mathrm{GH}$ emission, solar abundance should be expected. However, in the case where the soft excess is related to the Centaurus A structure, the abundance might be expected to be either sub-solar, if the X-ray-emitting gas is representative of the intergalactic medium interacting with the radio-emitting outflow (see, e.g., Siemiginowska et al. 2012), or even super-solar, if it is related to the matter uplifted by the expanding radio bubble from the Centaurus A host galaxy (e.g., Simionescu et al. 2008). The corresponding XIS spectra of the diffuse emission detected in the "Lobe 1" and "Lobe 2" regions are presented in Figure 9, together with the model curves $\left(Z=0.3 Z_{\odot}\right.$ fit).

To sum up, we conclude that the model we have applied provides a reasonable representation of the data, implying a relatively prominent soft X-ray excess at the position of Centaurus A giant lobes probed with Suzaku, which is best described as thermal emission from hot gas with a temperature of $k T \sim 0.5 \mathrm{keV}$. As already noted above, it may originate in the Centaurus A system, or instead may be due to some large-scale fluctuation in the foreground $\mathrm{GH}$ emission. The latter option cannot be ruled out with the available data (cf. the ROSAT map in Figure 7). However, in the discussion that follows (Section 3.1) we investigate the former possibility, arguing that the soft X-ray-emitting gas responsible for the observed excess is consistent with diffuse matter filling the lobe and mixed with the non-thermal plasma, or alternatively with a condensation of the intergalactic medium around the edges of the expanding radio structure. The estimated $0.5-2 \mathrm{keV}$ absorbed flux of this gas is roughly $\simeq 6 \times 10^{-13} \mathrm{erg} \mathrm{cm}^{-2} \mathrm{~s}^{-1} /$ $0.35 \mathrm{deg}^{2}$, meaning $\sim 2 \times 10^{-11} \mathrm{erg} \mathrm{cm}^{-2} \mathrm{~s}^{-1}$ for the entire halo $\left(\simeq 13.2 \mathrm{deg}^{2}\right)$, assuming that the "Lobe 1" and "Lobe 2" regions are indeed representative for the entire structure.

We finally note that the analysis of the data collected with the hard X-ray detector (PIN instrument) on board Suzaku (Takahashi et al. 2007) during the exposure that we carried out reveals no excess emission at the position of "Lobe 1 " and "Lobe 2" over the background. The implied 10-50 keV upper limit of $5 \times 10^{-12} \mathrm{erg} \mathrm{cm}^{-2} \mathrm{~s}^{-1} / 0.25 \mathrm{deg}^{2}$, or $F_{10-50 \mathrm{keV}}<$

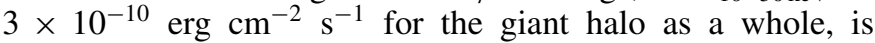
about one order of magnitude above the non-thermal diffuse flux in the $10-50 \mathrm{keV}$ range expected from the broadband modeling presented in Abdo et al. (2010), namely, $\sim 2 \times$ $10^{-11} \mathrm{erg} \mathrm{cm}^{-2} \mathrm{~s}^{-1}$. At lower photon energies, it is the level of the CXB component of the spectrum that provides the relevant upper limit for the non-thermal diffuse emission of the lobes. In detail, the total absorbed $2-10 \mathrm{keV}$ energy flux detected in the "on" pointings is $\simeq 6.5 \times 10^{-12} \mathrm{erg} \mathrm{cm}^{-2} \mathrm{~s}^{-1} /$ $0.35 \mathrm{deg}^{2}$ (see Table 5), basically as expected for the CXB surface brightness. This implies that any diffuse emission filling the halo should not exceed $\sim 10 \%$ of this value. Meanwhile, the 2-10 keV energy flux expected for the southern lobe of Centaurus A based on the Abdo et al. (2010) analysis is on average $\simeq 1.5 \times 10^{-13} \mathrm{erg} \mathrm{cm} \mathrm{cm}^{-2} \mathrm{~s}^{-1} / 0.35 \mathrm{deg}^{2}$, i.e., about a factor of four below the formal upper limit of $10 \%$ of the CXB level.

\section{DISCUSSION}

\subsection{Origin of the Diffuse Emission}

The results of the analysis of the Suzaku data regarding the diffuse emission positioned at the part of the southern giant lobe in the Centaurus A radio galaxy, as summarized above, suggest the presence of an excess component best modeled as thermal emission from a hot gas with temperature $k T \simeq 0.5 \mathrm{keV}$. Assuming that this excess originates within the Centaurus A halo rather than in the Galactic foreground, the returned MEKAL normalization (see Table 6),

$$
\text { Norm }=\frac{10^{-14}}{4 \pi D^{2}} \times\left(\frac{n_{g}}{\mathrm{~cm}^{-3}}\right)^{2} \times\left(\frac{\mathrm{ARF}}{\mathrm{cm}^{2}}\right) \times\left(\frac{\ell}{\mathrm{cm}}\right),
$$

where $\mathrm{ARF}=0.35 \mathrm{deg}^{2}$ with the conversion scale $1.08 \mathrm{kpc}$ $\operatorname{arcmin}^{-1}$ and $\ell$ is the third dimension of the emitting region, implies a number density for the X-ray-emitting gas of 

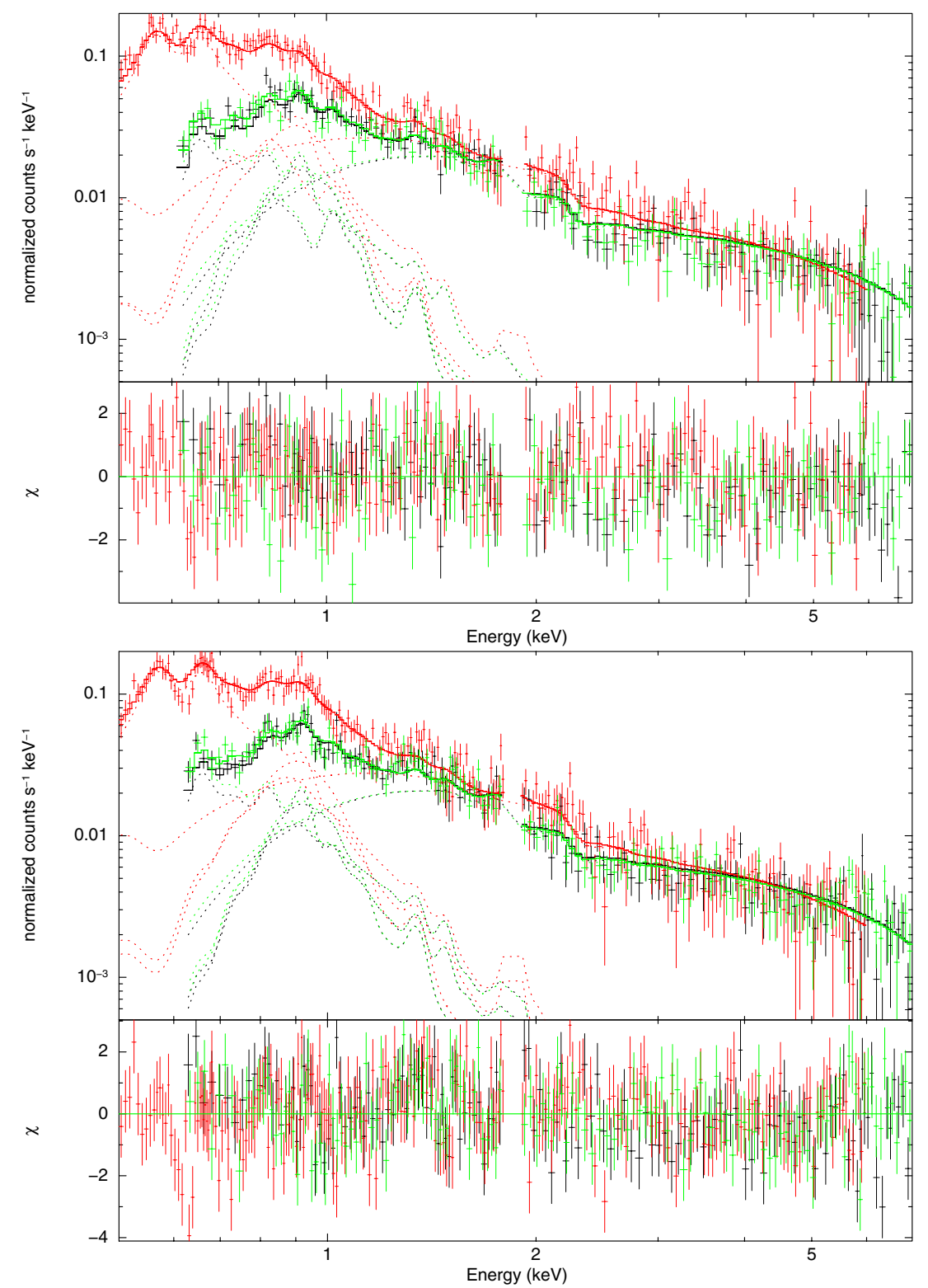

Figure 9. XIS spectra of the diffuse emission component detected in the "Lobe 1" and "Lobe 2" regions (upper and lower panels, respectively), together with the MEKAL+wabs* (MEKAL+PL+MEKAL) model curves corresponding to the $Z=0.3 Z_{\odot}$ fit described in Section 2.4. XIS0 data/fit are shown in black, XIS1 in red, and XIS3 in green.

$n_{g} \simeq(0.9-2.5) \times 10^{-4} \mathrm{~cm}^{-3}$, where we assume abundances within the range $10 \%-100 \%$ solar, and $\ell=150 \mathrm{kpc}$ as appropriate for gas that uniformly fills the entire southern lobe (see Hardcastle et al. 2009). Such a value for the density may be considered as relatively high, implying that the total mass of the thermal gas contained within the entire halo is of the order of $\bar{n}_{g} m_{p} V_{\ell} \sim 10^{10} M_{\odot}$ for the mean number density $\bar{n}_{g} \simeq$ $n_{g} \sim 10^{-4} \mathrm{~cm}^{-3}$ within the lobes' volume $V_{\ell} \simeq 2 \times 10^{71} \mathrm{~cm}^{-3}$. Yet if such a large amount of hot gas is indeed mixed with the diffuse non-thermal plasma, composed of a magnetic field with the volume-averaged field strength $\bar{B} \simeq 1 \mu \mathrm{G}$ roughly in pressure equipartition with the radio-emitting ultrarelativistic electrons-namely, $\left\langle U_{B}\right\rangle \equiv \bar{B}^{2} / 8 \pi \simeq\left\langle p_{e \pm}\right\rangle \equiv\left\langle U_{e \pm}\right\rangle / 3$ (Abdo et al. 2010) — then the lobes' thermal pressure $\left\langle p_{g}\right\rangle=$ $\bar{n}_{g} k \bar{T} \simeq 8 \times 10^{-14} \mathrm{erg} \mathrm{cm}^{-3}$ is almost exactly equal to the non-thermal one $\left\langle p_{e \pm}\right\rangle+\left\langle U_{B}\right\rangle \simeq 8 \times 10^{-14} \mathrm{erg} \mathrm{cm}^{-3}$. In other words, the sound velocity in the system $\bar{c}_{s}=\left(5 \mathrm{k} \bar{T} / 3 m_{p}\right)^{1 / 2} \simeq$ $3 \times 10^{7} \mathrm{~cm} \mathrm{~s}^{-1}$ is then approximately equal to the Alfvén ve- locity $\bar{v}_{A}=\bar{B} /\left(4 \pi m_{p} \bar{n}_{g}\right)^{1 / 2} \simeq 2 \times 10^{7} \mathrm{~cm} \mathrm{~s}^{-1}$, or equivalently the plasma parameter $\beta \equiv\left\langle p_{g}\right\rangle /\left\langle U_{B}\right\rangle \simeq\left(\bar{c}_{s} / \bar{v}_{A}\right)^{2} \sim 1$. Pressure equilibration between different plasma species in the giant lobes of Centaurus $\mathrm{A}$ is a meaningful characteristic, reminding us again of the interstellar medium (ISM) within the Galactic disk.

The numbers derived above challenge at the same time, at least to some extent, the alternative scenario for the soft $\mathrm{X}$-ray excess related to the Centaurus A system and involving a condensation of the hot intergalactic medium around the edges of the expanding radio structure. That is because in such a case the third dimension of the emitting volume is likely to be smaller than the scale of the lobes, ${ }^{21} \ell \ll 150 \mathrm{kpc}$, resulting

\footnotetext{
21 We note, however, that in the case of very light relativistic jets evolving in a stratified hot thermal atmosphere, it is possible to find a set of the model parameters for which the spatial scale of the gas compressed and heated by the bow shock driven by the expanding cocoon is comparable to the scale of the radio lobes (Hardcastle \& Krause 2013).
} 
in an increased gas number density $n_{g} \propto \ell^{-1 / 2}$ and therefore in an increased gaseous pressure $p_{g} \propto n_{g}$ largely exceeding $\left\langle p_{e \pm}\right\rangle+\left\langle U_{B}\right\rangle$. That is to say, this alternative scenario does not give self-consistent values for the model parameters, unless an additional pressure support is assumed for the lobes, for example, due to ultrarelativistic protons injected by the jets into the expanding cocoon (see, e.g., Ostrowski 2000). We also note in this context that the gas density in a typical poor group of galaxies at the distance $r$ from the group center, approximated by the standard profile

$$
n_{\text {igm }}(r) \simeq n_{0} \times\left(\frac{r}{a_{0}}\right)^{-b},
$$

with $r>a_{0} \simeq 10 \mathrm{kpc}, b \simeq 1.5$, and $n_{0} \lesssim 10^{-2} \mathrm{~cm}^{-3}$, yields the expected $n_{\text {igm }}(150 \mathrm{kpc}) \lesssim 10^{-4} \mathrm{~cm}^{-3}$; the relatively large scatter in the values of the parameters $a_{0}, b$, and $n_{0}$ found for various systems should be kept in mind (e.g., Mulchaey \& Zabludoff 1998; Sun 2012).

In general, the extended lobes in radio galaxies and quasars were previously believed to be largely devoid of thermal matter and to consist solely of relativistic particles and magnetic field deposited by a pair of jets over the entire lifetime of a source (e.g., Scheuer 1974; Begelman \& Cioffi 1989; Kino et al. 2012). More recently, this somewhat simplistic view has changed, as several observational findings triggered the discussion on possibly significant thermal content of the lobes in different systems. For example, observations of socalled double-double radio galaxies, i.e., the systems with inner/younger radio lobes evolving within and aligned with the outer/older lobes (Schoenmakers et al. 2000; Saikia \& Jamrozy 2009), suggested a mass density within the outer lobes in excess of that expected from a pure jet deposition. Kaiser et al. (2000) discussed in this context various mechanisms enabling the extended radio lobes to be enriched by the additional thermal matter, e.g., via uplifting of some part of the ISM from the galaxy host by the expanding outflow, as in fact observed recently in a few cases (Simionescu et al. 2008), or via the entrainment of warm and dense gaseous clouds present in the intergalactic medium by the lobes (followed by the clouds' destruction and dispersion over the entire lobe volume on the timescales of about $\sim 10 \mathrm{Myr}$ ). In addition, analysis of the pressure balance between the radio lobes and the surrounding intergalactic or intracluster medium for larger samples of objects led to the conclusion that the dominant pressure support in the lobes, in the case of the oldest and most evolved systems, cannot be entirely explained by the radio-emitting electrons and magnetic field, but in addition requires either thermal matter or a relativistic proton population (Hardcastle \& Worrall 2000; Dunn et al. 2005; Croston et al. 2008; Bîrzan et al. 2008). These are all strong yet indirect indications for the presence of thermal matter within the extended lobes of radio-loud AGNs. Until now, no direct evidence for such matter has been found in either X-ray or radio studies (Sanders \& Fabian 2007).

Upper limits for the thermal gas density within the lobes of luminous radio galaxies were previously derived from the apparent lack of any signatures of internal depolarization of the radio emission, with the often-claimed volume- and sampleaveraged product $\left\langle n_{g} \times B\right\rangle<5 \times 10^{-3} \mu \mathrm{G} \mathrm{cm}^{-3}$ (Garrington \& Conway 1991), meaning $\bar{n}_{g}<(1-10) \times 10^{-4} \mathrm{~cm}^{-3}$ for the typically derived field strength range $3 \mu \mathrm{G}<B<30 \mu \mathrm{G}$ (Kataoka \& Stawarz 2005; Croston et al. 2005; Isobe et al. 2011, and references therein). In the particular case of Centaurus A, the analysis of the improved RM data presented in O'Sullivan et al. (2013) reveals, for the very first time, strong indication for a positive detection of the internal depolarization signal, with a corresponding gas density $\bar{n}_{g} \sim 10^{-4} \mathrm{~cm}^{-3}$. This is in an excellent agreement with the results presented here based on a completely different data set. Such an agreement is rather encouraging, keeping in mind that the RM analysis by O'Sullivan et al. (2013) should be considered to be a more robust method for the detection of the thermal content of the Centaurus A giant lobes, as it probes the entire volume of the halo and not only a small part as in the case of the Suzaku data discussed here.

\subsection{Origin of Compact $X$-Ray Features}

In this section we return to the compact X-ray "spots" detected in our Suzaku observations and discuss their origin in more detail. In the estimates given below we evaluate plasma parameters corresponding to different emission models for a single spot with radius $R \leqslant 1.5 \simeq 1.6 \mathrm{kpc}$ producing a $0.5-10 \mathrm{keV}$ flux at the level of $F_{\mathrm{x}} \simeq 10^{-13} \mathrm{erg} \mathrm{cm}^{-2} \mathrm{~s}^{-1}$, i.e., characterized by an X-ray luminosity of, roughly, $L_{\mathrm{x}} \simeq$ $10^{38} \mathrm{erg} \mathrm{s}^{-1}$ (these parameters are roughly characteristic of the spots we observe; see Section 2.3).

\subsubsection{Bremsstrahlung Emission}

A thermal interpretation of the detected X-ray spots would imply the presence of compact overdensities in the gas distribution within the giant lobes of Centaurus A, with the temperatures $k T \simeq 5 \mathrm{keV}$ elevated by an order of magnitude with respect to the thermal diffuse pool characterized by the average $k \bar{T} \simeq 0.5 \mathrm{keV}$. Such overdensities could, in principle, constitute manifestations of the interactions of the magnetized radio-emitting outflow with pre-existing inhomogeneities in the ambient medium, as in fact observed in Centaurus A on somewhat smaller scales, around the middle and inner northern lobes, much closer to the active nucleus and the host galaxy (see Morganti 2010). There, the performed infrared, optical, and X-ray studies indicate the presence of dust clouds, filaments of young stars, or condensations of hot gas related to the radio structure and best explained in terms of interactions of the expanding jets/lobes in the system with the ambient matter, resulting in substantial heating of the plasma and the triggering of star formation (e.g., Kraft et al. 2009; Auld et al. 2012; Crockett et al. 2012). Similar phenomena are also observed in other analogous radio galaxies and hence seem generic for nearby, low-power radio-loud AGNs (e.g., Siemiginowska et al. 2012, and references therein).

In this context, radio lobes may be analogous to the Galactic center region, where the disturbed endpoints of prominent nonthermal radio filaments are observed to coincide with molecular clumps, consistent with the idea that magnetic energy is liberated as the large-scale magnetic field, tangled by the motion of the clumps, reconnects in the externally ionized surface layer of the advancing clumps (e.g., Staguhn et al. 1998; Sofue et al. 2005). This type of morphology reminds us to some extent of the complex structure in the southern giant lobe of Centaurus A revealed by our new X-ray and radio maps, suggesting a scenario in which the energy of the reconnecting magnetic field, released primarily in the form of short-scale magnetic turbulence, is used to heat the plasma of the interacting gaseous condensation and to form in this way the observed compact X-ray-emitting features, while also depolarizing the synchrotron radio emission of the filaments (cf. src 7 and 8 in Figure 6). 
In the framework of this model, the plasma energization timescale involved has to be shorter than the lifetime of the system, $\tau_{\text {life }} \sim 30$ Myr (Hardcastle et al. 2009), but not much shorter than the Coulomb collision timescale of the heated electron population in order to avoid any efficient acceleration of particles to higher (ultrarelativistic) energies and hence the formation of an energetically relevant non-thermal tail in the electron energy distribution (see the discussion in Petrosian \& East 2008). Assuming the "cold ambient plasma" regime for the Coulomb interactions, the appropriate collision timescale can be evaluated to be

$$
\tau_{\mathrm{Coul}} \simeq \frac{2 E_{e} v_{e}}{3 \sigma_{T} m_{e} c^{4} n_{g} \ln \Lambda} \sim 0.5 n_{-4}^{-1} \mathrm{Myr}
$$

for the Coulomb logarithm $\ln \Lambda \simeq 30$, electron kinetic energy $E_{e}=0.01 m_{e} c^{2}$ with the corresponding electron velocity $v_{e}$, and gas density $n_{g} \equiv n_{-4} \times 10^{-4} \mathrm{~cm}^{-3}$. It should be kept in mind, at the same time, that the "cold plasma regime" is not strictly valid for the electrons with $v_{e}$ close to the thermal velocities of the background particles, in which case the Coulomb collision timescale may be much longer than the one evaluated above (see Petrosian \& East 2008).

The problem with the above interpretation is, however, the energetics of the spots. The APEC normalization $\simeq(0.5-1) \times 10^{-4}$ derived for the analyzed src 5-8 (see Table 4) implies gas number densities $n_{g} \sim(3-5) \times 10^{-3} \mathrm{~cm}^{-3}$ for the assumed abundance $30 \%$ solar, the source extraction region $\pi R^{2}$, and the third dimension of the emitting volume $2 \times R$. In the limiting case of negligible metallicity, the number density of the gas with a temperature $k T \simeq 5 \mathrm{keV}$ needed to produce $10^{38} \mathrm{erg} \mathrm{s}^{-1}$ of thermal X-rays within a spot volume $V=(4 / 3) \pi R^{3}$ reads as

$$
n_{g} \simeq \sqrt{\frac{3 m_{e}^{3 / 2} c^{3} h(k T)^{1 / 2} L_{\mathrm{x}}}{32(2 \pi)^{1 / 2} e^{6} V f_{\mathrm{TB}}(T)}} \sim 6 \times 10^{-3} R_{1.5}^{-3 / 2} \mathrm{~cm}^{-3}
$$

where $R_{1.5} \equiv R / 1^{\prime} .5$ and

$$
f_{\mathrm{TB}}(T)=\int_{\varepsilon_{\min }}^{\varepsilon_{\max }} d \varepsilon \ln \left(\frac{4 k T}{1.78 \varepsilon}\right) \times \exp \left(-\frac{\varepsilon}{k T}\right),
$$

with $\varepsilon_{\min }=0.5 \mathrm{keV}$ and $\varepsilon_{\max }=10 \mathrm{keV}$. This is about two orders of magnitude above the number density of the diffuse thermal gas within the Centaurus A giant lobes, leading to a situation where the X-ray-emitting clumps are highly overpressured with respect to their surroundings, $n_{g} k T \sim$ $5 \times 10^{-11} R_{1.5}^{-3 / 2} \mathrm{erg} \mathrm{cm}^{-3} \gg\left\langle p_{g}\right\rangle$. One could speculate, on the other hand, that the compact features we observe are relatively short-lived, and that soon after the onset of a violent reconnection event in an isolated region within the lobe, followed by a rapid and efficient turbulent heating of the plasma in a confined volume, a newly formed overpressured spot disappears quickly on a sound-crossing timescale of, roughly, $\tau_{s} \simeq R / c_{s} \leqslant 2 R_{1.5}$ Myr. This, possibly, would correspond to the situation of a rather efficient transfer of the magnetic energy to the internal energy of the lobes' thermal plasma during the lifetime of the source.

\subsubsection{Inverse-Compton Emission}

One possibility for production of non-thermal X-ray emission from the spots is IC upscattering of cosmic microwave background $(\mathrm{CMB})$ photons (the dominant radiation field at the considered distance from the galactic host) by ultrarelativistic electrons with Lorentz factors $\gamma \simeq \sqrt{\varepsilon_{\mathrm{x}} / \varepsilon_{\mathrm{cmb}}} \sim 10^{3}$, where $\varepsilon_{\mathrm{x}} \simeq 1 \mathrm{keV}$ and the characteristic energy of the CMB radiation is $\varepsilon_{\mathrm{cmb}} \simeq 1 \mathrm{meV}$. The involved electron population could originate, for example, from a compression/re-acceleration of cosmic rays filling the giant lobes (and producing diffuse nonthermal emission at radio and $\mathrm{X}$-ray frequencies as modeled in Hardcastle et al. 2009; Abdo et al. 2010) by kpc-scale shocks formed around the reconnecting radio filaments.

Let us approximate the energy distribution of such a hypothetical additional electron population by an arbitrary form $d N_{e}(\gamma) / d \gamma \propto \gamma^{-3}$ between, say, $\gamma_{\min }=10$ and $\gamma_{\max }=10^{3}$, as very roughly justified by the $\mathrm{X}$-ray photon indices emerging from the PL fits to the spectra of the spots $(\Gamma \sim 2$ on average, albeit with a large spread). With such an assumption, the pressure of the electron population involved in producing the observed $\mathrm{X}$-ray emission is

$$
p_{e \pm} \simeq \frac{m_{e} c \gamma_{\min }^{-1} L_{\mathrm{x}}}{4 \sigma_{T} \ln \left(\frac{\gamma_{\max }}{\gamma_{\min }}\right) U_{\mathrm{cmb}} V} \sim 10^{-10} R_{1.5}^{-3} \mathrm{erg} \mathrm{cm}^{-3},
$$

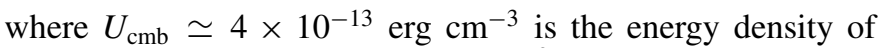
the CMB photon field, and we put $\int \gamma^{2} d N_{e}(\gamma) / \int \gamma d N_{e}(\gamma) \simeq$ $\ln \left(\gamma_{\max } / \gamma_{\min }\right) / \gamma_{\min }^{-1}$. Similarly to what we found in the case of a thermal model, this pressure exceeds by orders of magnitude the total pressure of the ambient plasma (especially for $R_{1.5}<1$ ), which invalidates the scenario drafted above and hence rules out an IC/CMB origin for the emission of the spots detected with Suzaku. The particular assumption regarding the exact form of the electron energy distribution used in the estimate above is not crucial in this respect, since even in the extreme case of a mono-energetic population of radiating electrons approximated by the delta function, $d N_{e}(\gamma) / d \gamma \propto \delta\left(\gamma-\gamma_{\max }\right)$, the corresponding electron pressure would be reduced only by a factor of $\ln \left(\gamma_{\max } / \gamma_{\min }\right) /\left(\gamma_{\max } / \gamma_{\min }\right) \simeq 0.04$, i.e., would still be very high, $\gg\left\langle p_{g}\right\rangle$.

\subsubsection{Synchrotron Emission}

The other non-thermal scenario to consider for the discussed $\mathrm{X}$-ray features is the synchrotron radiation of very high energy ultrarelativistic electrons. We note that synchrotron X-ray emission is routinely detected in blazars of the "high-frequency peaked BL Lac" type (e.g., Takahashi 2001), young supernova remnants (Vink 2012), kpc-scale jets in low-power radio galaxies (Harris \& Krawczynski 2006), or pulsar wind nebulae (Gaensler \& Slane 2006). In all these systems an efficient in situ acceleration of the X-ray-emitting electrons up to $\geqslant \mathrm{TeV}$ energies is required, although the exact energy dissipation processes involved, which in general are ill-understood, may be substantially different in different classes of objects. The particular multiwavelength morphology revealed by our maps, with some of the compact X-ray spots coinciding with extended and highly polarized radio filaments, prompts us to consider in this context the scenario where the magnetic energy released at the locations of reconnecting magnetic tubes/radio filaments is responsible for the confinement and acceleration of relativistic particles via a Fermi process within distinct emission sites (Drake et al. 2006; Hoshino 2012).

Let us therefore consider this possibility more quantitatively, in the analogy to the turbulent acceleration scenario developed in the context of solar flares (see Petrosian 2012, and references therein). For this we simply assume that within the volume, which may be approximated as spherical with radius $R$, 


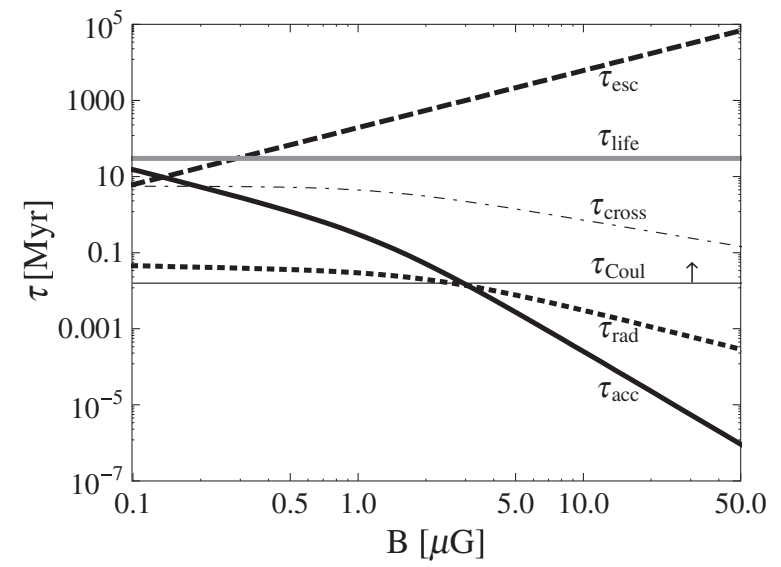

Figure 10. Acceleration, cooling, and escape timescales for ultrarelativistic electrons emitting synchrotron X-rays, as functions of the magnetic field $B$ within the considered emission site. The thick dotted curve denotes the radiative loss timescale, the thick solid curve corresponds to the acceleration timescale, and the thick dashed curve illustrates the escape timescale for an emission region size $R=1.6 \mathrm{kpc}$. We also plot the age of the Centaurus A giant lobes (horizontal gray line), the magnetosonic crossing timescale (thin dot-dashed curve), and the lower limit for the Coulomb collision timescale for the background thermal plasma (thin horizontal line).

small-scale magnetic turbulence is efficiently injected around the reconnecting large-scale magnetic field $B$, so that the total turbulent energy is roughly comparable to the magnetic energy, $(\delta B / B)^{2} \sim 1$. With such strong turbulence conditions, the mean free path of ultrarelativistic electrons for particle-wave interactions is expected to be of the order of the electron gyroradius, $\lambda_{e \pm} \sim r_{e \pm}=\gamma m_{e} c^{2} / e B \ll R$, and the spatial diffusion coefficient is $\kappa_{e \pm} \sim \lambda_{e \pm} c / 3$. Hence, the timescale for the particle escape from the acceleration site $\tau_{\text {esc }}(\gamma) \sim R^{2} / \kappa_{e \pm}$. The spatial diffusion of the electrons is obviously accompanied by their diffusion in momentum space, leading to a net acceleration at the maximum allowed rate with the characteristic timescale $\tau_{\mathrm{acc}}(\gamma) \sim \lambda_{e \pm} c / v_{\mathrm{sc}}^{2}$, where $v_{\mathrm{sc}}$ is the velocity of the interacting waves (Alfvén or fast magnetosonic waves in the case of ultrarelativistic electrons). The evolving electrons also undergo radiative energy losses, dominated in our case by the synchrotron and IC/CMB processes; the characteristic timescale for these can be approximated as $\tau_{\text {rad }}(\gamma) \sim 3 m_{e} c / 4 \sigma_{T} \gamma U_{0}$, where $U_{0}(\gamma)=U_{B}+U_{\mathrm{cmb}} f_{\mathrm{KN}}(\gamma), U_{B} \equiv B^{2} / 8 \pi$, and $f_{\mathrm{KN}}(\gamma)=$ $\left(1+4 \gamma \varepsilon_{\mathrm{cmb}} / m_{e} c^{2}\right)^{-3 / 2}$ stands for the Klein-Nishina correction to the IC scattering cross section. The synchrotron scenario can therefore be considered as a viable option if the maximum available ("equilibrium") electron energy $\gamma_{\text {eq }}$ given by the condition $\tau_{\text {acc }}\left(\gamma_{\text {eq }}\right) \sim \tau_{\text {rad }}\left(\gamma_{\text {eq }}\right) \ll \tau_{\text {esc }}\left(\gamma_{\text {eq }}\right)$ is large enough to produce synchrotron photons with $\varepsilon_{\mathrm{x}}=3 h e B \gamma^{2} / 4 \pi m_{e} c \sim 1 \mathrm{keV}$ energies. This is indeed possible to achieve for a magnetic field only slightly larger than the volume-averaged value, $B \gtrsim 1 \mu \mathrm{G}$ $\simeq \bar{B}$, as in fact would be expected for highly polarized filaments (Feain et al. 2011).

In Figure 10 we plot all the analyzed timescales corresponding to $\gamma_{\mathrm{eq}}$ electrons emitting synchrotron $\varepsilon_{\mathrm{x}}=1 \mathrm{keV}$ photons as functions of the magnetic field $B$ within the considered region of radius $R$. The thick dotted curve denotes the radiative loss timescale. As shown, synchrotron losses dominate over the IC/CMB process for $B \gtrsim$ a few $\mu \mathrm{G}$. The thick solid curve in the figure corresponds to the acceleration timescale with the velocities of the turbulent modes set to be $v_{\mathrm{sc}}=\sqrt{v_{A}^{2}+c_{s}^{2}}$, where the Alfvén velocity $v_{A}$ is evaluated for a background gas density $\bar{n}_{g}=10^{-4} \mathrm{~cm}^{-3}$, while the sound velocity $c_{s}$ assumes a background gas temperature $k \bar{T}=0.5 \mathrm{keV}$. It follows that the Alfvénic acceleration dominates for $B>1 \mu \mathrm{G}$. The thick dashed curve shows the escape timescale of the $\mathrm{X}$-ray-emitting electrons, $\tau_{\mathrm{esc}}>100 \mathrm{Myr}$ for $R=1.6 \mathrm{kpc}$ and $B>1 \mu \mathrm{G}$. For comparison, we also plot the estimated lifetime 22 of the giant halo $\tau_{\text {life }} \simeq 30 \mathrm{Myr}$ (thick horizontal gray line), the magnetosonic crossing timescale $\tau_{\text {cross }} \simeq R / v_{\text {sc }}$ with $R=1.6 \mathrm{kpc}$ and $v_{s} c$ as above (thin dot-dashed curve), and the lower limit ("cold ambient plasma" regime; see equation) for the Coulomb collision timescale $\tau_{\text {Coul }} \simeq 0.01 \mathrm{Myr}$ of the background thermal gas (thin horizontal line); these are all longer than $\tau_{\text {acc }}$ and $\tau_{\text {rad }}$ for $B \gtrsim$ a few $\mu \mathrm{G}$. Figure 10 indicates, therefore, that, assuming strong turbulent conditions, efficient acceleration and confinement of the radiating electrons within compact and substantially magnetized emission sites are plausible.

Importantly, for the particular conditions of the emission site discussed here, namely, a very inefficient particle escape when compared with the acceleration and radiative loss timescales (and also the lifetime of the system), as well as the stochastic nature of the acceleration process, the resulting electron population is expected to be of a "modified ultrarelativistic Maxwellian" form $d N_{e}(\gamma) / d \gamma \propto \gamma^{2} \exp \left[-(1 / a)\left(\gamma / \gamma_{\mathrm{eq}}\right)^{a}\right]$ with the parameter $0<a<2$ depending on particular cooling and turbulence conditions, as discussed in Stawarz \& Petrosian (2008), i.e., to be peaked (with a broader plateau) around the equilibrium energy $\gamma_{\mathrm{eq}}$. The high-energy segment of the synchrotron continuum produced by such electrons seems to be qualitatively consistent with the observed spectral properties of the analyzed X-ray spots (see Figure 12 in Stawarz \& Petrosian 2008), while at lower (radio-to-optical) frequencies very flat synchrotron continua would be expected. For such an electron population, $\int \gamma^{2} d N_{e}(\gamma) / \int \gamma d N_{e}(\gamma) \sim \gamma_{\mathrm{eq}}$, and the total pressure of the discussed X-ray-emitting electron population is expected to be rather tiny,

$$
p_{e \pm} \simeq \frac{m_{e} c L_{\mathrm{x}}}{4 \sigma_{T} \gamma_{\mathrm{eq}} U_{B} V} \sim 2 \times 10^{-16} B_{\mu \mathrm{G}}^{-3 / 2} R_{1.5}^{-3} \mathrm{erg} \mathrm{cm}^{-3}
$$

where $B_{\mu \mathrm{G}} \equiv B / \mu \mathrm{G}$, so that it starts to exceed the thermal pressure of the surrounding medium $\left\langle p_{g}\right\rangle$ or the magnetic pressure $U_{B}$ within the emission site only for $R$ significantly smaller than hundreds of parsecs. This is the advantage of the synchrotron scenario over the previous two considered above. The interesting aspect of the model is the implied production of very high energy $\gamma$-rays $\left(\varepsilon_{\gamma} \gtrsim 10 \mathrm{TeV}\right)$ by the synchrotron $\mathrm{X}$-ray-emitting electrons via the accompanying IC/CMB process; the total diffuse $\gamma$-ray flux thus expected for the entire giant halo can be evaluated very roughly as $F_{\gamma \text {, tot }} \simeq$ $\left(U_{\mathrm{cmb}} / U_{B}\right) \times F_{\mathrm{X}, \text { tot }} \sim 10^{-11} \mathrm{erg} \mathrm{cm}^{-2} \mathrm{~s}^{-1}$ for the spots' magnetic field of the order of a few $\mu \mathrm{G}$.

\footnotetext{
22 The lifetime estimates of Hardcastle et al. (2009) are derived on the assumption of no efficient in situ particle acceleration in the lobe, and so the use of this lifetime estimate in this context is not strictly self-consistent; we retain it for simplicity, noting that significant particle acceleration would tend to artificially reduce lifetimes estimated with spectral ageing methods, and also that in the framework of the discussed model very efficient electron acceleration is restricted only to compact turbulent regions of the lobes, which we identify as sites of violent reconnection of large-scale magnetic tubes/radio filaments, and is not distributed within the whole volume of the giant halo.
} 


\section{SUMMARY AND CONCLUSIONS}

In this paper we have discussed Suzaku observations of selected regions within the southern giant lobe of the Centaurus A radio galaxy. In the analysis we have focused first on distinct X-ray features detected with the XIS instrument, at least some of which are likely associated with fine structure of the lobe revealed by the most recent high-quality radio intensity and polarization maps. We found that from the available photon statistics, the spectral properties of the detected X-ray features are equally consistent with thermal emission from hot gas with temperatures $k T \simeq 1-10 \mathrm{keV}$, or with power-law radiation continua characterized by photon indices $\Gamma \simeq 2.0 \pm 0.5$. The plasma parameters implied by these different models favor a synchrotron origin of the analyzed X-ray spots, which indicates that a very efficient acceleration of electrons up to $\gtrsim 10 \mathrm{TeV}$ energies is taking place within the giant structure of Centaurus A, albeit only in isolated and compact regions associated with extended and highly polarized radio filaments. We speculate that the magnetic energy released locally at the sites of reconnecting magnetic tubes/radio filaments loads turbulence into the system, and that this turbulence is in turn responsible for the confinement and acceleration of relativistic particles within distinct emission sites. Alternatively, if most of the released magnetic energy goes into heating of the plasma rather than acceleration of ultrarelativistic particles, the thermal scenario, involving short-lived and highly overpressured gaseous clumps, remains a valid possibility, suggesting an efficient transfer of the magnetic energy to the internal energy of the lobes' thermal plasma during the lifetime of the source.

Large-scale highly polarized radio filaments have been detected within the lobes of several other famous radio galaxies (Fomalont et al. 1989; Carilli et al. 1991; Perley et al. 1997; Swain et al. 1998; Owen et al. 2000). Apparently, similar structures are observed in the Galactic center region (Lang et al. 1999; LaRosa et al. 2000; Nord et al. 2004; Yusef-Zadeh et al. 2004). The origin and the exact structure of such features are unclear, but tangled magnetic field tubes are often invoked in this context (e.g., O’Neill \& Jones 2010). The X-ray observations reported here constitute an interesting insight into the problem, suggesting in particular that, as in fact expected in the case of tangled field tubes by analogy with solar phenomena, localized regions of enhanced turbulence enable efficient cosmic-ray acceleration and/or plasma heating form a set of transient "hot spots" in compact sites where separate filaments interact with each other or with overdensities in the ambient plasma so that magnetic reconnection takes place.

While we have emphasized the possibility of reconnection acting as a source of the turbulence, there are other processes that could account for such a localized enhancement. If the Centaurus A giant halo is indeed a source of very high to ultrahigh energy cosmic rays, as has been discussed in the literature (Hardcastle et al. 2009; O'Sullivan et al. 2009; Pe'er \& Loeb 2012) prompted by the results of the P. Auger Observatory (e.g., Moskalenko et al. 2009; Nemmen et al. 2010; Yüksel et al. 2012), the flux of these particles through such regions can in principle produce uncompensated currents, amplifying small-scale magnetic fields and turbulent motions, in a process analogous to that frequently considered in the context of supernova remnants (Bell 2004).

We have also presented a detailed analysis of the diffuse emission component filling the whole FOV of the XIS instrument, which remains after removing all the compact X-ray features.
We found that at the position of the Centaurus A giant lobes probed with Suzaku a relatively prominent soft X-ray excess, best described as thermal emission from hot gas with the temperature of $k T \simeq 0.5 \mathrm{keV}$, seems to be present. This excess, if related to the Centaurus A system rather than some large-scale fluctuation in the foreground $\mathrm{GH}$ emission, may be either due to thermal matter mixed with the non-thermal plasma of the giant lobes or due to a condensation of the hot intergalactic medium around the edges of the expanding radio structure. The former possibility would interestingly imply a number density of the X-ray-emitting gas of roughly $n_{g} \simeq 10^{-4} \mathrm{~cm}^{-3}$, as well as almost exact pressure balance between the lobes' thermal and non-thermal contents (again in analogy with the ISM within the Galactic disk). These conclusions, which are still tentative and somewhat preliminary, are in agreement with the most recent analysis of the radio data presented by O'Sullivan et al. (2013). It is important to emphasize that our finding regarding the plasma $\beta$ parameter being of the order of unity in the giant lobes of Centaurus A may be crucial for understanding the dynamics and evolution of the reconnecting magnetic field in the system. Gourgouliatos \& Lyutikov (2012) argued, for example, that the evolution of the expanding lobes containing some thermal plasma and some magnetic flux will always be accompanied by a decrease in the $\beta$ parameter, leading at some point to the spontaneous formation of electric current sheets and large-scale reconnection layers, which are likely involved in acceleration of the highest energy cosmic rays.

Regardless of the exact processes involved in the formation of the observed X-ray sub-structure of the lobes, however, or of the origin of the soft diffuse excess emission, the total $\mathrm{X}$-ray emission of the whole giant halo in Centaurus A appears to be a complex mixture of diffuse and compact thermal and non-thermal components. Only with the broad energy coverage and the suitable combination of the sensitivity and resolution of Suzaku has it been possible to disentangle these components; otherwise, they would form a single emission continuum. If our preliminary conclusion that these various emission components are indeed related to the Centaurus A system is correct, there are important implications for understanding the physics of radioloud AGNs: the structure of the extended lobes in such systems may be highly inhomogeneous and non-uniform, with magnetic reconnection and turbulent acceleration processes continuously converting magnetic energy to the internal energy of the plasma particles, leading to possibly significant spatial and temporal variations in the plasma $\beta$ parameter and pressure equilibrium conditions. Forthcoming Suzaku pointings and the follow-up Chandra observations of the Centaurus A giant lobes will shortly allow us to test the above conclusions.

Finally, we note that a possibly significant contribution from compact features and thermal plasma to the total observed X-ray flux of the extended lobes means that without detailed goodquality X-ray maps enabling the various emission components to be disentangled, the ratio of total X-ray and radio fluxes, typically used to infer volume-averaged magnetic field intensity (under the working assumption that the IC scattering of CMB photons by radio-emitting electrons uniformly filling the lobe provides the dominant contribution to the measured X-ray flux), should be used with some caution (see the related discussion in Hardcastle et al. 2007). In particular, departures from the energy equipartition, often claimed because of apparently high values of this ratio (e.g., Kataoka \& Stawarz 2005; Croston et al. 2005; Isobe et al. 2011, and references therein), may be partly invalidated, and the lobes, instead of being dominated by the 
Table 7

Alternative Modeling Results for the Diffuse Emission

\begin{tabular}{|c|c|c|c|c|c|c|c|c|}
\hline $\begin{array}{l}\text { Region } \\
\text { (1) }\end{array}$ & $\begin{array}{c}Z_{\mathrm{GH}} / Z_{\odot} \\
\quad(2)\end{array}$ & $\begin{array}{c}k T_{\mathrm{GH}} \\
(3)\end{array}$ & $\begin{array}{c}\operatorname{Norm}_{G H} \\
\text { (4) }\end{array}$ & $\begin{array}{c}Z_{\text {ext }} / Z_{\odot} \\
\quad(5)\end{array}$ & $\begin{array}{c}k T_{\text {ext }} \\
(6)\end{array}$ & $\begin{array}{c}\text { Norm }_{\text {ext }} \\
\text { (7) }\end{array}$ & $\begin{array}{c}\Gamma_{\mathrm{CXB}} \\
(8)\end{array}$ & $\begin{array}{c}\text { Norm }_{C X B} \\
(9)\end{array}$ \\
\hline Lobe 1 (“on”) & $1.0^{f}$ & $0.16_{-0.02}^{+0.01}$ & $\begin{array}{l}7.34_{-1.52}^{+3.48} \\
\end{array}$ & $0.3^{f}$ & $0.59 \pm 0.02$ & $3.60 \pm 0.17$ & $1.41^{f}$ & $1.04 \pm 0.02$ \\
\hline Lobe 2 (“on”) & $"$ & $"$ & $"$ & $"$ & $0.73 \pm 0.02$ & $3.15 \pm 0.15$ & $"$ & $"$ \\
\hline Lobe 3 (“off”) & $"$ & " & $"$ & $"$ & $0.71 \pm 0.11$ & $1.43 \pm 0.27$ & $"$ & $1.30_{-0.03}^{+0.04}$ \\
\hline Lobe 4 (“off”) & ” & $"$ & $"$ & " & $1.59 \pm 0.36$ & $1.31 \pm 0.30$ & $"$ & $"$ \\
\hline
\end{tabular}

Notes. (1) Region fitted; (2) fixed (" $f$ ”) abundance of the GH component; (3) temperature of the GH component in keV units; (4) APEC normalization of the GH component $\times 10^{-3}$; (5) fixed (" $f$ ") abundance of the extra thermal component; (6) temperature of the extra thermal component in keV units; (7) APEC normalization of the extra thermal component $\times 10^{-3}$; $(6)$ fixed (" $f$ ") photon index of the CXB component; (9) normalization of the CXB component $\times 10^{-3}$.

relativistic particle pressure, may well be on average very close to energy equipartition, $\left\langle p_{g}\right\rangle \sim\left\langle p_{e \pm}\right\rangle \sim\left\langle U_{B}\right\rangle$, as proposed in this paper for the Centaurus A giant lobes.

Ł.S. and M.O. were supported by Polish NSC grant DEC2012/04/A/ST9/00083. S.O'S. acknowledges the support of the Australian Research Council through grant FL100100033. Work by C.C.C. at NRL is supported in part by NASA DPR S15633-Y. B.R. has received funding from the European Research Council under the European Community's Seventh Framework Programme (FP7/2007-2013)/ERC grant agreement no. 247039.

\section{APPENDIX}

As a cross-check of the modeling of the diffuse emission component in all four analyzed Suzaku pointings (see Section 2.4), we fit the diffuse spectra obtained after removing all the point sources present in the FOVs with the same model wabs $*$ (APEC_1 + APEC_2 + PL) including an absorbed thermal component (APEC_1) representing GH emission, an absorbed power-law component corresponding to the $\mathrm{CXB}$ radiation, and an additional absorbed thermal component (APEC_2) describing any residual thermal emission related to the Centaurus A system.

We utilized the data in the $0.5-7 \mathrm{keV}$ photon energy range for XIS1 and 0.6-7 keV range for XIS0/3 and applied $C$-statistics (preferred over the $\chi^{2}$-statistics in the case of limited counts) in the simultaneous spectral fitting. The neutral hydrogen column density was fixed as before at the Galactic value in the direction of the source, $N_{\mathrm{H}, \mathrm{Gal}}=0.7 \times 10^{21} \mathrm{~cm}^{-2}$. For the $\mathrm{GH}$ component, we fixed the abundance at the solar value and required the temperature and the normalization to be the same in all four spectra. The photon index for the CXB component was fixed as $\Gamma_{\mathrm{CXB}}=1.41$ as before, but the CXB normalization was allowed to vary between "Lobes 1 and 2" and "Lobes 3 and 4" spectra. This choice was dictated by the rather different net exposures of the "on" and "off" pointings, and the consequently different flux levels of the point sources removed, which in particular affects the high-energy segment of the spectra. Finally, the metallicity of the extra thermal component was fixed at a value of 0.3 solar, but its temperature and normalization were allowed to vary between all pointings. The results of the fitting are summarized in Table 7. As shown, an excess $\simeq 0.6 \mathrm{keV}$ emission at the position of the lobes is recovered just as before, with the normalization of the APEC_2 component in the "on" pointings being about twice as high as in the "off" pointings (cf. Table 5).

\section{REFERENCES}

Abdo, A. A., Ackermann, M., Ajello, M., et al. 2010, Sci, 328, 725 Aharonian, F., Akhperjanian, A. G., Anton, G., et al. 2009, ApJL, 695, L40
Arp, H. 1994, A\&A, 288, 738

Auld, R., Smith, M. W. L., Bendo, G., et al. 2012, MNRAS, 420, 1882

Beckmann, V., Jean, P., Lubiński, P., Soldi, S., \& Terrier, R. 2011, A\&A, 531, A70

Begelman, M. C., \& Cioffi, D. F. 1989, ApJL, 345, L21

Bell, A. R. 2004, MNRAS, 353, 550

Bîrzan, L., McNamara, B. R., Nulsen, P. E. J., Carilli, C. L., \& Wise, M. W. 2008, ApJ, 686, 859

Carilli, C. L., Perley, R. A., Dreher, J. W., \& Leahy, J. P. 1991, ApJ, 383, 554

Cooper, B. F. C., Price, R. M., \& Cole, D. J. 1965, AuJPh, 18, 589

Crockett, R. M., Shabala, S. S., Kaviraj, S., et al. 2012, MNRAS, 421, 1603

Croston, J. H., Hardcastle, M. J., Birkinshaw, M., Worrall, D. M., \& Laing, R. A. 2008, MNRAS, 386, 1709

Croston, J. H., Hardcastle, M. J., Harris, D. E., et al. 2005, ApJ, 626, 733

Drake, J. F., Swisdak, M., Che, H., \& Shay, M. A. 2006, Natur, 443, 553

Dunn, R. J. H., Fabian, A. C., \& Taylor, G. B. 2005, MNRAS, 364, 1343

Feain, I. J., Cornwell, T. J., Ekers, R. D., et al. 2011, ApJ, 740, 17

Feain, I. J., Ekers, R. D., Murphy, T., et al. 2009, ApJ, 707, 114

Fomalont, E. B., Ebneter, K. A., van Breugel, W. J. M., \& Ekers, R. D 1989, ApJL, 346, L17

Foster, A. R., Ji, L., Smith, R. K., \& Brickhouse, N. S. 2012, ApJ, 756, 128

Fukazawa, Y., Hiragi, K., Yamazaki, S., et al. 2011, ApJ, 743, 124

Gaensler, B. M., \& Slane, P. O. 2006, ARA\&A, 44, 17

Gandhi, P., \& Fabian, A. C. 2003, MNRAS, 339, 1095

Gandhi, P., Horst, H., Smette, A., et al. 2009, A\&A, 502, 457

Garrington, S. T., \& Conway, R. G. 1991, MNRAS, 250, 198

Georgakakis, A., Nandra, K., Laird, E. S., Aird, J., \& Trichas, M. 2008, MNRAS, 388,1205

Gourgouliatos, K. N., \& Lyutikov, M. 2012, MNRAS, 420, 505

Hardcastle, M. J., Cheung, C. C., Feain, I. J., \& Stawarz, Ł. 2009, MNRAS, 393, 1041

Hardcastle, M. J., Croston, J. H., \& Kraft, R. P. 2007, ApJ, 669, 893

Hardcastle, M. J., \& Krause, M. G. H. 2013, MNRAS, 430, 174

Hardcastle, M. J., \& Worrall, D. M. 2000, MNRAS, 319, 562

Harris, D. E., \& Krawczynski, H. 2006, ARA\&A, 44, 463

Hoshino, M. 2012, PhRvL, 108, 135003

Ishihara, D., Onaka, T., Kataza, H., et al. 2010, A\&A, 514, A1

Ishisaki, Y., Maeda, Y., Fujimoto, R., et al. 2007, PASJ, 59, 113

Isobe, N., Makishima, K., Tashiro, M., \& Kaneda, H. 2001, in ASP Conf. Proc. 250, Particles and Fields in Radio Galaxies Conference, ed. R. A. Laing \& K. M. Blundell (San Francisco, CA: ASP), 394

Isobe, N., Seta, H., \& Tashiro, M. S. 2011, PASJ, 63, 947

Israel, F. P. 1998, A\&ARv, 8, 237

Jarrett, T. H., Cohen, M., Masci, F., et al. 2011, ApJ, 735, 112

Johnson, S. P., Dong, H., \& Wang, Q. D. 2009, MNRAS, 399, 1429

Junkes, N., Haynes, R. F., Harnett, J. I., \& Jauncey, D. L. 1993, A\&A, 269, 29

Kaiser, C. R., Schoenmakers, A. P., \& Rottgering, H. J. A. 2000, MNRAS, 315,381

Kataoka, J., \& Stawarz, Ł. 2005, ApJ, 622, 797

Katsuta, J., Tanaka, Y. T., Stawarz, L., et al. 2013, A\&A, 550, A66

Kim, D.-W. 2012, Hot Interstellar Matter in Elliptical Galaxies (Astrophysics and Space Science Library, Vol. 378; Berlin: Springer), 121

Kino, M., Kawakatu, N., \& Takahara, F. 2012, ApJ, 751, 101

Koyama, K., Tsunemi, H., Dotani, T., et al. 2007, PASJ, 59, 23

Kraft, R. P., Forman, W. R., Hardcastle, M. J., et al. 2009, ApJ, 698, 2036

Lang, C. C., Anantharamaiah, K. R., Kassim, N. E., \& Lazio, T. J. W. 1999, ApJL, 521, L41

LaRosa, T. N., Kassim, N. E., Lazio, T. J. W., \& Hyman, S. D. 2000, AJ, 119, 207

Lehmer, B. D., Xue, Y. Q., Brandt, W. N., et al. 2012, ApJ, 752, 46 
Lu, F. J., Yuan, T. T., \& Lou, Y.-Q. 2008, ApJ, 673, 915

Marshall, F. J., \& Clark, G. W. 1981, ApJ, 245, 840

Mitsuda, K., Bautz, M., Inoue, H., et al. 2007, PASJ, 59, 1

Morganti, R. 2010, PASA, 27, 463

Moskalenko, I. V., Stawarz, L., Porter, T. A., \& Cheung, C. C. 2009, ApJ, 693, 1261

Mulchaey, J. S., \& Zabludoff, A. I. 1998, ApJ, 496, 73

Nemmen, R. S., Bonatto, C., \& Storchi-Bergmann, T. 2010, ApJ, 722, 281

Neumayer, N. 2010, PASA, 27, 449

Nord, M. E., Lazio, T. J. W., Kassim, N. E., et al. 2004, AJ, 128, 1646

O’Neill, S. M., \& Jones, T. W. 2010, ApJ, 710, 180

Ostrowski, M. 2000, MNRAS, 312, 579

O’Sullivan, S. P., Feain, I. J., McClure-Griffiths, N. M., et al. 2013, ApJ, 764, 162

O’Sullivan, S., Reville, B., \& Taylor, A. M. 2009, MNRAS, 400, 248

Owen, F. N., Eilek, J. A., \& Kassim, N. E. 2000, ApJ, 543, 611

Pe'er, A., \& Loeb, A. 2012, JCAP, 3, 7

Perley, R. A., Roser, H.-J., \& Meisenheimer, K. 1997, A\&A, 328, 12

Petrosian, V. 2012, SSRv, 173, 535

Petrosian, V., \& East, W. E. 2008, ApJ, 682, 175

Saikia, D. J., \& Jamrozy, M. 2009, BASI, 37, 63

Sakano, M., Warwick, R. S., Decourchelle, A., \& Predehl, P. 2003, MNRAS, 340,747

Sanders, J. S., \& Fabian, A. C. 2007, MNRAS, 381, 1381

Scheuer, P. A. G. 1974, MNRAS, 166, 513

Schoenmakers, A. P., de Bruyn, A. G., Rottgering, H. J. A., van der Laan, H., \& Kaiser, C. R. 2000, MNRAS, 315, 371
Serlemitsos, P. J., Soong, Y., Chan, K.-W., et al. 2007, PASJ, 59, 9

Siemiginowska, A., Stawarz, Ł., Cheung, C. C., et al. 2012, ApJ, 750, 124

Simionescu, A., Werner, N., Finoguenov, A., Böhringer, H., \& Brüggen, M. 2008, A\&A, 482, 97

Sofue, Y., Kigure, H., \& Shibata, K. 2005, PASJ, 57, L39

Soifer, B. T., Neugebauer, G., \& Houck, J. R. 1987, ARA\&A, 25, 187

Staguhn, J., Stutzki, J., Uchida, K. I., \& Yusef-Zadeh, F. 1998, A\&A, 336, 290

Stawarz, Ł., \& Petrosian, V. 2008, ApJ, 681, 1725

Steinle, H., Bennett, K., Bloemen, H., et al. 1998, A\&A, 330, 97

Sun, M. 2012, NJPh, 14, 045004

Swain, M. R., Bridle, A. H., \& Baum, S. A. 1998, ApJL, 507, L29

Takahashi, T. 2001, in AIP Conf. Ser. 558, High Energy Gamma-Ray Astronomy, ed. F. A. Aharonian \& H. J. Voelk (Melville, NY: AIP), 346

Takahashi, T., Abe, K., Endo, M., et al. 2007, PASJ, 59, 35

Takeuchi, Y., Kataoka, J., Stawarz, Ł., et al. 2012, ApJ, 749, 66

Tawa, N., Hayashida, K., Nagai, M., et al. 2008, PASJ, 60, 11

Tozzi, P., Rosati, P., Nonino, M., et al. 2001, ApJ, 562, 42

Ueda, Y., Akiyama, M., Ohta, K., \& Miyaji, T. 2003, ApJ, 598, 886

Vink, J. 2012, A\&ARv, 20, 49

Wright, E. L., Eisenhardt, P. R. M., Mainzer, A. K., et al. 2010, AJ, 140,1868

Yamamura, I., Makiuti, S., Ikeda, N., et al. 2010, yCat, 2298, 0

Yuasa, T., Nakazawa, K., \& Makishima, K. 2009, PASJ, 61, 1107

Yüksel, H., Stanev, T., Kistler, M. D., \& Kronberg, P. P. 2012, ApJ, 758, 16

Yusef-Zadeh, F., Hewitt, J. W., \& Cotton, W. 2004, ApJS, 155, 421 